\title{
Control of Virulent Listeria monocytogenes Originating from Dairy Products and Cattle Environment Using Marine Algal Extracts, Silver Nanoparticles Thereof, and Quaternary Disinfectants
}

Mona A El-Zamkan,' Bassma A Hendy, ${ }^{2}$ Hassan Mahmoud Diab, ${ }^{3}$ Najat Marraiki, ${ }^{4}$ Gaber El-Saber Batiha, ${ }^{5}$ Hani Saber, ${ }^{6}$ Waleed Younis, (iD) ${ }^{7}$ Shankar Thangamani, ${ }^{8}$ Khalid J Alzahrani, (iD) ${ }^{9}$ Ahmed Shaban Ahmed'

'Department of Food Hygiene and Control (Milk Hygiene), Faculty of Veterinary Medicine, South Valley University, Qena, 83523, Egypt; ${ }^{2}$ Reference Lab for Food Safety, Animal Health Research Institute (AHRI), Agriculture Research Center (ARC), Dokki, Giza, 12622, Egypt; ${ }^{3}$ Department of Animal and Poultry Health and Environment, Faculty of Veterinary Medicine, South Valley University, Qena, 83523, Egypt; ${ }^{4}$ Department of Botany and

Microbiology, College of Science, King Saud University, Riyadh, I I45I, Saudi Arabia; ${ }^{5}$ Department of Pharmacology and Therapeutics, Faculty of Veterinary Medicine, Damanhour University, Damanhour, Al-Beheira 225I I, Egypt; ${ }^{6}$ Department of Botany and Microbiology, Faculty of Science, South Valley University, Qena, 83523, Egypt; ${ }^{7}$ Department of Microbiology, Faculty of Veterinary Medicine, South Valley University, Qena, 83523, Egypt; ${ }^{8}$ Department of Pathology and Population Medicine, College of Veterinary Medicine, Midwestern University, Glendale, AZ, USA; ${ }^{9}$ Department of Clinical Laboratories Sciences, College of Applied Medical Sciences, Taif University, Taif, 21944, Saudi Arabia

Correspondence: Mona A El-Zamkan Department of Food Hygiene and Control (Milk Hygiene), Faculty of Veterinary Medicine, South Valley University, Qena, 83523, Egypt

Email m_zam@vet.svu.edu.eg

Shankar Thangamani

Department of Pathology and Population Medicine, College of Veterinary Medicine, Midwestern University, Glendale, AZ, USA Email sthang@midwestern.edu
Introduction: Listeria monocytogenes is an important foodborne pathogen of public- and animalhealth concern globally. The persistence of L. monocytogenes in the dairy-processing environment has multifactorial causes, including lack of hygiene, inefficient cleaning, and improper disinfection practices.

Materials and Methods: A total of 300 dairy-product and environmental samples were collected from dairy-cattle facilities and local dairy shops and vendors in Qena, Egypt. Samples were screened for the incidence of Listeria spp. and to detect virulence determinants and disinfectant-resistance genes. Three marine algal species - Caulerpa racemosa, Jania rubens, and Padina pavonica - were collected from Hurghada on the Red Sea coast. Algal extracts were screened using gas chromatography-mass spectrometry. The antimicrobial activity of some marine algal extracts, nanoparticles derived therefrom, and some disinfectants against $L$. monocytogenes strains were assessed in vitro using agar-well diffusion and liquid-broth methods. The impact of $P$. pavonica extract on the growth and survival of virulent $L$. monocytogenes in cheese and whey were clarified.

Results and Discussion: The incidence of L. monocytogenes in dairy products and environmental samples was $15.5 \%$ and $19 \%$, respectively. The most common toxigenic gene profile found among the isolates was $h l y A^{+}-i n l A^{+}-p r f A^{+}$. The sensitivity pattern of $L$. monocytogenes strains to disinfectant containing alkyl $\left(\mathrm{C}_{12-16}\right)$ dimethyl BAC was high compared to other tested quaternary ammonium compounds (QAC) disinfectants tested, which showed lower log reductions against resistant strains. The QAC disinfectant-resistance gene $q a c H$ was detected in $40 \%$ of the isolates. Potent bactericidal activity of a petroleum ether extract of $P$. pavonica and silver nanoparticles of $P$. pavonica were obtained against the virulent $L$. monocytogenes strain. The population of $L$. monocytogenes in cheese curd and whey after 14 days was reduced at a rate of $9 \log \mathrm{CFU} / \mathrm{g}$ and $8 \log$ $\mathrm{CFU} / \mathrm{mL}$, respectively due to the effect of $P$. pavonica extract. After 28 days of storage, $L$. monocytogenes was completely inactivated in those dairy products.

Conclusion: P. pavonica extract showed promising antimicrobial properties, calling for further comprehensive studies prior to it being applied in the food industry to enhance the safety, quality, and shelf life of products and protect public health.

Keywords: Listeria monocytogenes, virulence genes, cheese, qacH gene, disinfectants, algae, antimicrobial activity

\section{Introduction}

The genus Listeria is ubiquitous in nature and occurs frequently on farms and in food processing, handling, and storage environments. ${ }^{1}$ L. monocytogenes causes 
listeriosis, which is one of the most serious foodborne diseases in humans, especially in pregnant women, neonates, and immunocompromised adults. ${ }^{2}$

The virulence potential of $L$. monocytogenes relies on several molecular determinants, including virulence genes responsible for the invasion of host cells ( $i n l A$, inlB, and iap), phagosomal escape ( $h l y A, p l c A$, and $p l c B)$ and positive regulatory factor A $(\operatorname{Prf} A){ }^{3}$ All these facilitate the intracellular growth and spread of the bacterium within a mammalian host. ${ }^{4}$ Another trait of L. monocytogenes is its colonization on working surfaces and transmission to dairy products, ${ }^{5}$ even after cleaning and disinfection, due to subinhibitory concentrations of disinfectants on different scales. $^{6}$ Disinfectants based on quaternary ammonium compounds (QACs) are widely used in the food industry, and are known to be effective against L. monocytogenes. ${ }^{7}$ Tolerance to such QACs as benzalkonium chloride (BAC) has been observed in many L. monocytogenes strains. Multiple genetic markers have been identified that confer L. monocytogenes resistance to QACs, including the $q a c H$ gene of transposon Tn6188. ${ }^{8}$

Chemical or physical preservatives have traditionally been used to prevent L. monocytogenes degradation of food causing microbial resistance and the emergence of human- and animal-health risks. ${ }^{9,10}$ Biopreservation is an alternative technologies that enhances food safety, stability, and quality using natural additives, and has gained increased attention. ${ }^{11,12}$ Marine algae could prove to be promising agents to replace synthetic antimicrobial agents used in the food industry, because of their substantial natural bioactive compounds with broad antimicrobial bioactivity. ${ }^{13}$ Antimicrobial action of marine algae against foodborne pathogens, including L. monocytogenes, has been confirmed. ${ }^{14}$ As such, utilization of algal extracts and nanoparticle (NP) derivatives thereof as antimicrobial agents for food preservation could be an interesting alternative to physical and chemical methods.

As raw milk and ready-to-eat food products, including soft or semisoft cheese, are majorly involved in listeriosis outbreaks, ${ }^{15,16}$ and the ability of L. monocytogenes to resist hostile conditions, including low temperature, osmotic stress, high salt content, and mild preservation treatment, its ability to form biofilms, ${ }^{17}$ and the antimicrobial and antioxidant properties of microalga-derived compounds that have been used as food ingredients, ${ }^{12,14}$ the present study aimed to investigate the incidence of Listeria spp., especially L. monocytogenes, in dairy-product (cheese and dairy desserts) and dairy-cattle environmental samples in Qena, Egypt, and the presence of some virulence genes in the obtained L. monocytogenes isolates, detect $L$. monocytogenes resistance to some surface disinfectants and molecular detection of the $q a c H$ resistance gene, and control L. monocytogenes propagation in both the dairy environment and ripened soft white cheese using some marine algal extracts, NP derivatives thereof, and QACs disinfectants.

\section{Materials and Methods}

\section{Ethics Approval}

Ethics approval was not required for this study.

\section{Collection of Samples}

A total of 300 dairy-product (200) and environmental (100) samples were collected from dairy-cattle facilities and local dairy shops and vendors in Qena. From dairy shops and vendors, 25 milk-product samples were collected: cheese (feta, kareish, talaga, processed) and dairy desserts (ice cream, custard, mahalabia, rice milk). Fifty drinking-water troughs and udder water-wash samples were collected as per the $\mathrm{WHO},{ }^{18}$ as well as 25 cattlebedding and 25 manure samples as per Rendos et al. ${ }^{19}$

\section{Isolation and Identification of Listeria Species}

Isolation of Listeria spp. was carried out as per Roberts and Greenwood $^{20}$ using Listeria-selective enrichment broth (CM0862, Oxoid) supplemented with the Listeriaselective enrichment agent SR0141 (Oxoid) and Oxford Listeria-selective agar (CM0856, Oxoid) supplemented with the Listeria-selective supplement SR0140 (Oxoid). Presumptive Listeria spp. isolates were confirmed according to Gram reactions and biochemical identification. ${ }^{21}$ The isolated and characterized L. monocytogenes strains were confirmed using a Microbact Listeria 12L kit system (Oxoid) as per Chye et al. ${ }^{22}$

\section{Detection of Virulence and Disinfectant- Resistance Gene qacH in L. monocytogenes by PCR}

Isolated $L$. monocytogenes strains underwent PCR to detect $h l y \mathrm{~A}$, inlA, and prfA virulence genes, while disinfectant resistance in $L$. monocytogenes isolates was detected using $q a c \mathrm{H}$. Primer sequences and amplicon size are presented in Table 1. ${ }^{23,24}$ Equipment and material used comprised a QIAamp DNA mini kit (51304), 


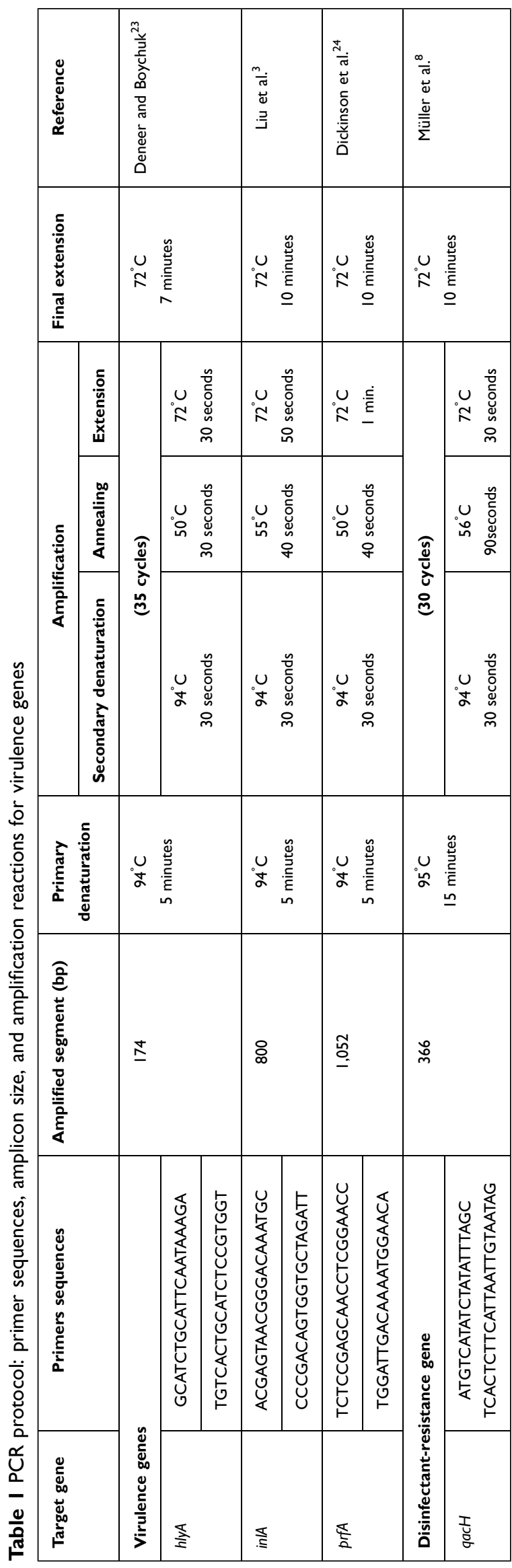

DreamTaq green PCR master mix $(2 \times), \mathrm{K} 1081$; Thermo FisherScientific), and agarose-gel electrophoresis. ${ }^{25}$

\section{Evaluation of Antibacterial Activity of Disinfectants Against L. monocytogenes}

$\mathrm{W}$ aimed to evaluate the bactericidal activity of three disinfectants - BAC (Sigma-Aldrich), Vanoquat (alkyl [c $\mathrm{c}_{12-16}$ ] dimethyl BAC, $\mathrm{c}_{12-15}$ alcohol ethoxylate; Evans Vanodine International), and BioSentry 904 QAC base (Hacco) - against L. monocytogenes strains.

Testing was performed as per European Norm 1276, ${ }^{26}$ which specifies a quantitative suspension test for evaluation of bactericidal activity of chemical disinfectants used in the food industry. Bacterial suspensions were prepared from fresh L. monocytogenes strains that had been isolated. The bacterial $\log$ count was adjusted to $9 \log _{10}$ using spectrophotometry and McFarland standards. The mixture was maintained at $20^{\circ} \mathrm{C}$ for 2 minutes. Then, the disinfectant solutions were added and the mixtures maintained at $20^{\circ} \mathrm{C}$ for 5 and 10 minutes. After this, an aliquot was taken and the bactericidal activity in this portion immediately neutralized or suppressed by the dilution-neutralization method using a mixture of polysorbate $8030.0 \mathrm{~g} / \mathrm{L}$, saponin $30 \mathrm{~g} / \mathrm{L}$, lecithin $3 \mathrm{~g} / \mathrm{L}$, and histidine $1 \mathrm{~g} / \mathrm{L}$ for QAC-based disinfectants. ${ }^{27,28}$ After neutralization for 5 minutes at $20^{\circ} \mathrm{C}, 1 \mathrm{~mL}$ of the neutralized test mixture was immediately taken and diluted with diluent to $10^{-7}$ dilution. The $\log$ count of $L$. monocytogenes was determined after plate counting on specific media.

\section{Antibacterial Activity of Algal Extracts and Nanoparticle Derivatives Against $L$. monocytogenes \\ Algal Collection and Extraction Preparation}

Three marine algal species - Caulerpa racemosa (Chlorophyta), Jania rubens (Rhodophyta), and Padina pavonica (Phaeophyta) - were collected from Hurghada on the Red Sea coast during March 2019. These species were identified according to standard taxonomic keys ${ }^{29,30}$ Samples were collected in sterilized polyethylene bags and kept in an icebox for transport to the laboratory. Sample preparation and extraction were done as per to Ahmed et al. $^{31}$ The weighted crude extracts were suspended in dimethyl sulfoxide (DMSO) to a final concentration of $50 \mathrm{mg} / \mathrm{mL}$, then stored in airtight bottles in a refrigerator until used for experiments. 


\section{Analysis of Algal Extracts}

Algal extracts were analyzed using gas chromatographymass spectrometry. technique. A Thermo Fisher Scientific Trace 1310 with capillary column TG-5 $(30 \times 250 \times 0.25$ $\mu \mathrm{m})$ was used. A mass detector was used in split mode, and helium gas with flow rate $1.5 \mathrm{~mL} / \mathrm{min}$ was used as a carrier. The injector was operated at $230^{\circ} \mathrm{C}$ and oven temperature for initial setup was $60^{\circ} \mathrm{C}$ for 2 minutes, ramping up by $10^{\circ} \mathrm{C} / \mathrm{min}$ to $300^{\circ} \mathrm{C}$ for 8 minutes. Mass spectra were taken at $70 \mathrm{eV}$, and total $\mathrm{GC}$ run time was 35 minutes.

\section{Biosynthesis and Characterization of Silver} Nanoparticles of $P$. pavonica (Ag-NPs-P. pavonica) Biosynthesis and characterization of Ag-NPs-P. pavonica were performed: $1 \mathrm{~g}$ algal powder was dissolved in $100 \mathrm{~mL}$ distilled water in a $250 \mathrm{~mL}$ Erlenmeyer flask and heated in a water bath at $60^{\circ} \mathrm{C}$ for 1 hour. The heated extract was filtered through Whatman No. 1 filter paper. The filtrates were collected, and the algal extracts were stored in fridge at $4^{\circ} \mathrm{C}$. Ag-NPs were synthesized by adding $10 \mathrm{~mL}$ of pure algal extracts to $90 \mathrm{~mL}$ of $\mathrm{AgNO}_{3}(1 \mathrm{mM})$ in a $250 \mathrm{~mL}$ Erlenmeyer flask, heated in water bath at $60^{\circ} \mathrm{C}$ for 1 hour with continuous stirring, then incubated in the dark for 24 hours at room temperature. $^{31}$

\section{Characterization of Ag-NPs-P. pavonica}

Characterization of Ag-NPs was performed as described previously by Saber et $\mathrm{al}^{32}$ and El-Sheekh et al. ${ }^{33} \mathrm{Ag}-\mathrm{NP}$ morphology was measured with transmission electron microscopy (TEM) JEOL 2010. Dynamic light scattering (DLS) was used for measuring size distribution, and potential was assessed using Zetasizer Nano compact scattering (Malvern Instruments).

\section{Anti-L. monocytogenes Activity of Algal Extracts and Ag-NPs-P. pavonica}

The anti-L. monocytogenes activity of algae extracts and Ag-NPs- $P$. pavonica against virulent and QAC-resistant strains was detected using agar-well diffusion as per Zainol et al $^{34}$ and the liquid-broth method described by Ahmed et al. ${ }^{31}$ In the latter method, data are expressed as $\log \mathrm{CFU} / \mathrm{mL}$ as a function of alga-extract concentration in the assay medium. All assays were performed in triplicate, and the results are given as means of three independent experiments.

\section{Impact of $P$. pavonica Extract on Growth and Survival of Virulent L. monocytogenes in Soft Cheese and Whey}

The initial suspension of L. monocytogenes was prepared using the $0.5 \mathrm{McF}$ arland standard. The strain used was carrying all the virulence and disinfectant-resistance genes for which it was being investigated. Cheese was prepared at the lab from UHT milk warmed to $40^{\circ} \mathrm{C}$ and then $5 \% \mathrm{NaCl}$ and rennet extract $(1: 6,000)$ added. Cheese was manufactured as per Abou-Donia, ${ }^{35}$ with some modification. Then, an inoculum of $L$. monocytogenes was added to obtain a load of $5 \log$ $\mathrm{CFU} / \mathrm{mL}$. After thorough mixing of the milk-bacteria suspension, P. pavonica extract was added at a concentration of 750 $\mu \mathrm{g} / \mathrm{mL}$. The mixture was left to coagulate for $2-3$ hours at $40^{\circ}$ $\mathrm{C}$, then whey was removed completely from the curd. Portions $(1 \mathrm{~g}$ and $1 \mathrm{~mL}$ ) were taken from the curd and whey, respectively, at once to enumerate L. monocytogenes, and counting was done for the first 3 days and then weekly during the ripening period. The manufactured cheese was stored at $10^{\circ}$ $\mathrm{C}$ in soldered tins. L. monocytogenes was enumerated by surface plating using Oxford Listeria-selective agar supplemented with Listeria-selective supplement. Cheese kept as control was manufactured and examined with the same procedure without addition of the algal extract. Results are expressed as CFU/g. Logarithmic bacterial load reduction was calculated:

$\log _{10}\left[\frac{A}{B}\right]$

where $A$ is the L. monocytogenes count in cheese kept as control and $B$ the $L$. monocytogenes count in cheese with $P$. pavonica extract, both in CFU/g.

\section{Results}

\section{Incidence of $L$. monocytogenes Isolated} from Dairy Products and Environmental Samples

Overall $38 \%, 45 \%$ and $46 \%$ of samples yielded Listeria spp.: cheese, dairy desserts, and environmental samples, respectively. Of these $13 \%, 18 \%$, and $19 \%$ of samples were positive for L. monocytogenes, respectively. From the 100 cheese samples, $16 \%, 20 \%, 16 \%$, and 0 of feta, kareish, talaga, and processed cheese were contaminated with $L$. monocytogenes, while $L$. monocytogenes was isolated from $20 \%, 16 \%, 8 \%$, and $28 \%$ of ice cream, custard, mahalabia, and rice milk samples, respectively (Table 2). Among the tested environmental samples, $46 \%$ were positive for Listeria spp., of which $41 \%$ were identified as $L$. monocytogenes. Manure samples contaminated with 


\begin{tabular}{|c|c|c|c|c|c|c|c|c|c|c|c|c|c|c|c|c|c|c|}
\hline \multicolumn{2}{|l|}{$a$} & $\frac{\text { 옹 }}{0}$ & 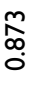 & $\begin{array}{l}\text { ஸ̊ } \\
\text { ஸุ } \\
0\end{array}$ & 오 & 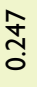 & $\frac{\infty}{\circ}$ & $\underset{0}{\stackrel{N}{\Upsilon}}$ & $\frac{\Lambda}{\infty}$ & $\begin{array}{l}\text { ̊̊ } \\
\text { ஸे }\end{array}$ & $\frac{m}{0}$ & 荬 & $\frac{\text { 으 }}{0}$ & $\underset{\stackrel{\sim}{*}}{\stackrel{\sim}{*}}$ & $\begin{array}{l}\bar{\wp} \\
\infty \\
0\end{array}$ & $\begin{array}{l}\text { ஸे } \\
\stackrel{+}{\circ}\end{array}$ & $\stackrel{\text { 응 }}{\circ}$ & ㅇㅇㅇ \\
\hline \multicolumn{2}{|l|}{ 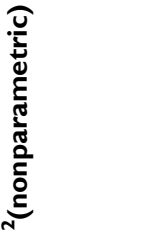 } & $\frac{\hat{\sigma}}{i}$ & $\stackrel{\bar{m}}{\stackrel{\sim}{ }}$ & 이 & 0 & $\begin{array}{l}\overline{\text { }} \\
\text { in }\end{array}$ & $\underset{+}{\stackrel{\infty}{N}}$ & $\frac{\infty}{\sim}$ & 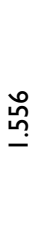 & 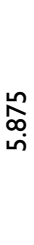 & $\begin{array}{l}\text { ல̊ } \\
\text { İ }\end{array}$ & $\begin{array}{l}\text { f. } \\
\underline{-}\end{array}$ & 우ํ & 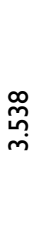 & 우 & $\underset{m}{\stackrel{\infty}{N}}$ & $\stackrel{\stackrel{m}{m}}{\underline{m}}$ & 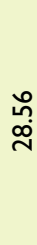 \\
\hline \multirow{2}{*}{ 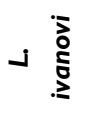 } & ১ீ & $\nabla$ & $\infty$ & $\nabla$ & $\nabla$ & Ln & $\nabla$ & $\nabla$ & $\nabla$ & $\infty$ & Ln & Ln & $\underline{\simeq}$ & $\infty$ & $\infty$ & $\nabla$ & $\infty$ & 0 \\
\hline & $\dot{0}$ & - & $N$ & - & - & Ln & - & - & - & $N$ & n & 으 & $m$ & $N$ & $N$ & - & $\infty$ & $\underline{\infty}$ \\
\hline \multirow{2}{*}{ ن } & ১ீ & $\infty$ & $\underline{\simeq}$ & $\infty$ & $\nabla$ & $\infty$ & $\stackrel{\circ}{\circ}$ & $\infty$ & $\nabla$ & $\infty$ & Ln & กٌ & $\infty$ & $\simeq$ & $\nabla$ & $\nabla$ & $\lambda$ & ๑ \\
\hline & : & $N$ & $m$ & $N$ & - & $\infty$ & 0 & $N$ & - & $N$ & เn & $\underline{m}$ & $N$ & $m$ & - & - & $\lambda$ & 유 \\
\hline \multirow{2}{*}{ 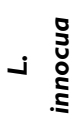 } & ১゚ & $\underline{\simeq}$ & $\infty$ & $\nabla$ & $\stackrel{\circ}{\circ}$ & 0 & $\nabla$ & $\infty$ & $\infty$ & $\underline{\simeq}$ & $\infty$ & $\Lambda$ & $\nabla$ & $\infty$ & $\infty$ & $\nabla$ & $\bullet$ & ف) \\
\hline & $\stackrel{\circ}{Z}$ & $m$ & $N$ & - & 0 & $\bullet$ & - & $N$ & $N$ & $m$ & $\infty$ & \pm & - & $N$ & $N$ & - & $\bullet$ & 유 \\
\hline \multirow{2}{*}{ 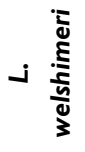 } & ১ீ & $\infty$ & $\nabla$ & $\infty$ & $\nabla$ & 0 & $\infty$ & $\infty$ & $\simeq$ & $\infty$ & $\sigma$ & $\stackrel{n}{\sim}$ & $\underline{\simeq}$ & $\nabla$ & 0 & $\infty$ & 0 & $\Lambda$ \\
\hline & i & $N$ & - & $N$ & - & $\bullet$ & $N$ & $N$ & $m$ & $N$ & $\sigma$ & $\underline{\underline{n}}$ & $m$ & - & 0 & $N$ & $\bullet$ & $\bar{\sim}$ \\
\hline \multirow{2}{*}{ 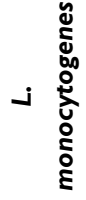 } & ১゚ & $\underline{0}$ & 오 & $\underline{0}$ & $\stackrel{\circ}{\circ}$ & $\underline{m}$ & 유 & $\underline{0}$ & $\infty$ & $\stackrel{\infty}{\sim}$ & $\underline{\infty}$ & ํㅜㅁ & $\stackrel{\infty}{\sim}$ & 오 & $\underline{\simeq}$ & $\underline{0}$ & 으 & $\stackrel{0}{\underline{0}}$ \\
\hline & í & $\nabla$ & เ & $\nabla$ & 0 & $\underline{m}$ & Ln & $\nabla$ & $N$ & $\lambda$ & $\underline{\infty}$ & $\bar{m}$ & $\Lambda$ & in & $m$ & $\nabla$ & $\underline{a}$ & 윰 \\
\hline \multirow{2}{*}{ 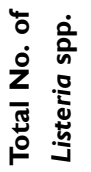 } & ১ீ & $\stackrel{\infty}{+}$ & กี & 우 & $\simeq$ & $\stackrel{\infty}{m}$ & $\stackrel{m}{m}$ & \& & లి & ठु & $\stackrel{\text { ํ }}{+}$ & $\frac{\Perp n}{\dot{\gamma}}$ & ఫु & กี & $\stackrel{\sim}{m}$ & $\stackrel{\text { m }}{ }$ & 우 & $\stackrel{m}{+}$ \\
\hline & $\dot{2}$ & $\simeq$ & $\underline{m}$ & 으 & $m$ & $\stackrel{\infty}{m}$ & $\sigma$ & 二 & $\sigma$ & $\underline{0}$ & $\stackrel{\sim}{\sim}$ & $\cong$ & $\underline{0}$ & $\underline{m}$ & $\infty$ & $\sigma$ & $\stackrel{\circ}{+}$ & సิ \\
\hline
\end{tabular}
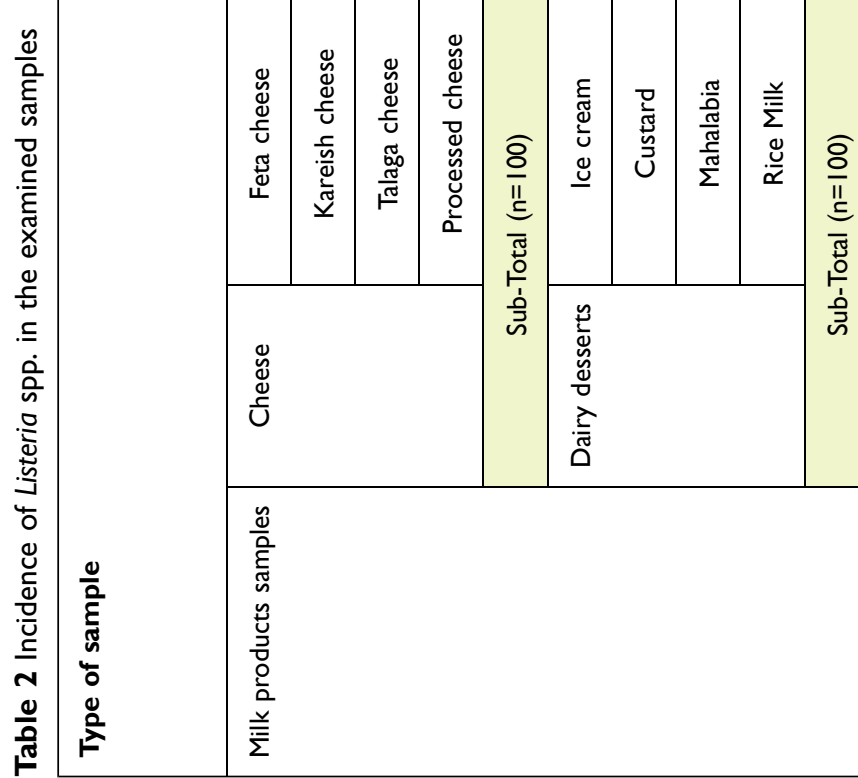

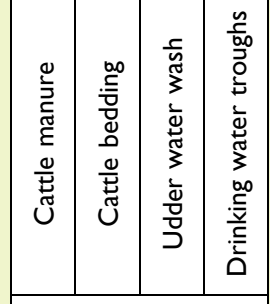


Listeria spp. had incidence of $64 \%$ and $28 \%$ pathogenic $L$. monocytogenes strains, which represented about $43.8 \%$ of Listeria spp. Furthermore, Listeria spp. existed in about $36 \%$ of drinking water-trough samples, and $44.4 \%$ of these isolates were found to be L. monocytogenes (Table 2 and Table S1). Other Listeria isolates were identified as L. welshimeri $(7 \%)$, L. innocua $(6.7 \%)$, L. seeligeri (6.7\%), and L. ivanovi (6\%; Table 2).

\section{Incidence of Virulence Genes in Isolated L. monocytogenes}

$h l y A$, inlA, and prfA were found in $54.8 \%, 67.7 \%$, and $61.3 \%$, respectively, of $L$. monocytogenes isolates of dairy products and $52.6 \%, 73.7 \%$, and $57.9 \%$ in environmental samples, respectively (Table 3 and Figure 1). Specific virulence-gene profiles turned out to be more common than others in the current study. Eight virulence-gene profiles were detected in our survey, which covered a total of 50 isolates. The most common virulence profile found among the isolates was toxin profile 1 $\left(h l y A^{+} / i n l A^{+} / p r f A^{+}\right)$, where strains isolated from most examined samples were found, followed by toxin profile $4\left(i n l A^{+} / p r f A^{+}\right)$and toxin profile $2\left(h l y A^{+} /\right.$inl $\left.A^{+}\right)$. Other profiles are shown in Figure 2 and Table S2. In almost all profiles, isolated strains in cheese, dairy desserts, and environmental samples were involved.

\section{Antibacterial Activity of Disinfectants and QAC-Resistance Genes}

All strains of L. monocytogenes (50) isolated from dairy and environmental samples were screened for susceptibility to/ resistance against QAC disinfectants. The overall prevalence of susceptible strains was 30 of 50 (60\%): 20 isolates from dairy products and the remaining ten from environmental samples. A total of $20 \mathrm{~L}$. monocytogenes isolates showed resistance against QAC disinfectant: eleven isolated from dairy products and nine isolates recovered from environmental samples.

Table 3 Virulence-gene profile of $L$. monocytogenes strains screened by $P C R^{\mathrm{a}}$

\begin{tabular}{|c|c|c|c|c|c|c|c|c|c|c|}
\hline \multicolumn{3}{|c|}{ Type of sample } & \multicolumn{2}{|c|}{ hlyA } & \multicolumn{2}{|c|}{ inIA } & \multicolumn{2}{|c|}{ prfA } & \multirow{3}{*}{$\begin{array}{c}\mathbf{P} \\
\text { value } \\
0.667\end{array}$} & \multirow{3}{*}{$\begin{array}{c}{ }^{2} \text { (nonparametric) } \\
0.717\end{array}$} \\
\hline & & & \multirow{2}{*}{$\begin{array}{c}\text { No. } \\
2\end{array}$} & \multirow{2}{*}{$\begin{array}{l}\% \\
50\end{array}$} & \multirow{2}{*}{$\frac{\text { No. }}{4}$} & \multirow{2}{*}{$\begin{array}{c}\% \\
100\end{array}$} & \multirow{2}{*}{$\begin{array}{c}\text { No. } \\
3\end{array}$} & \multirow{2}{*}{\begin{tabular}{|l|}
$\%$ \\
75
\end{tabular}} & & \\
\hline $\begin{array}{l}\text { Milk products } \\
\text { samples }\end{array}$ & Cheese & $\begin{array}{l}\text { Feta cheese } \\
\qquad(n=4)\end{array}$ & & & & & & & & \\
\hline & & Kareish cheese $(n=5)$ & 3 & 60 & 3 & 60 & 4 & 80 & 0.200 & 0.905 \\
\hline & & Talaga cheese $(n=4)$ & 2 & 50 & 2 & 50 & 3 & 75 & 0.286 & 0.867 \\
\hline & \multicolumn{2}{|c|}{ Sub-Total $(n=13)$} & 7 & 53.8 & 9 & 69.2 & 10 & 76.9 & 0.538 & 0.764 \\
\hline & \multirow{4}{*}{$\begin{array}{c}\text { Dairy } \\
\text { desserts }\end{array}$} & Ice cream $(n=5)$ & 3 & 60 & 3 & 60 & 2 & 40 & 0.250 & 0.883 \\
\hline & & Custard $(n=4)$ & 2 & 50 & 2 & 50 & 3 & 75 & 0.286 & 0.867 \\
\hline & & Mahalabia $(n=2)$ & 1 & 50 & 2 & 100 & I & 50 & 0.500 & 0.779 \\
\hline & & Rice Milk (n=7) & 4 & 57.1 & 5 & 71.4 & 3 & 42.9 & 0.500 & 0.779 \\
\hline & \multicolumn{2}{|c|}{ Sub-Total $(n=18)$} & 10 & 55.6 & 12 & 66.7 & 9 & 50 & 0.452 & 0.798 \\
\hline \multicolumn{3}{|l|}{ Total $(n=31)$} & 17 & 54.8 & 21 & 67.7 & 19 & 61.3 & 0.421 & 0.810 \\
\hline \multirow{4}{*}{\multicolumn{2}{|c|}{ Water and environmental samples }} & Cattle manure $(n=7)$ & 3 & 42.9 & 4 & 57.1 & 5 & 71.4 & 0.500 & 0.779 \\
\hline & & Cattle bedding $(n=5)$ & 4 & 80 & 4 & 80 & 2 & 40 & 0.800 & 0.670 \\
\hline & & Udder water wash $(n=3)$ & 1 & 33.3 & 2 & 66.7 & I & 33.3 & 0.500 & 0.779 \\
\hline & & $\begin{array}{l}\text { Drinking water troughs } \\
\qquad(\mathrm{n}=4)\end{array}$ & 2 & 50 & 4 & 100 & 3 & 75 & 0.667 & 0.717 \\
\hline \multicolumn{3}{|l|}{ Total $(n=19)$} & 10 & 52.6 & 14 & 73.7 & 11 & 57.9 & 0.743 & 0.690 \\
\hline \multicolumn{3}{|c|}{ Overall Total $(n=50)$} & 27 & 54 & 35 & 70 & 30 & 60 & 1.065 & 0.587 \\
\hline
\end{tabular}

Notes: ${ }^{a}$ All processed cheese samples were devoid of $L$. monocytogenes. 


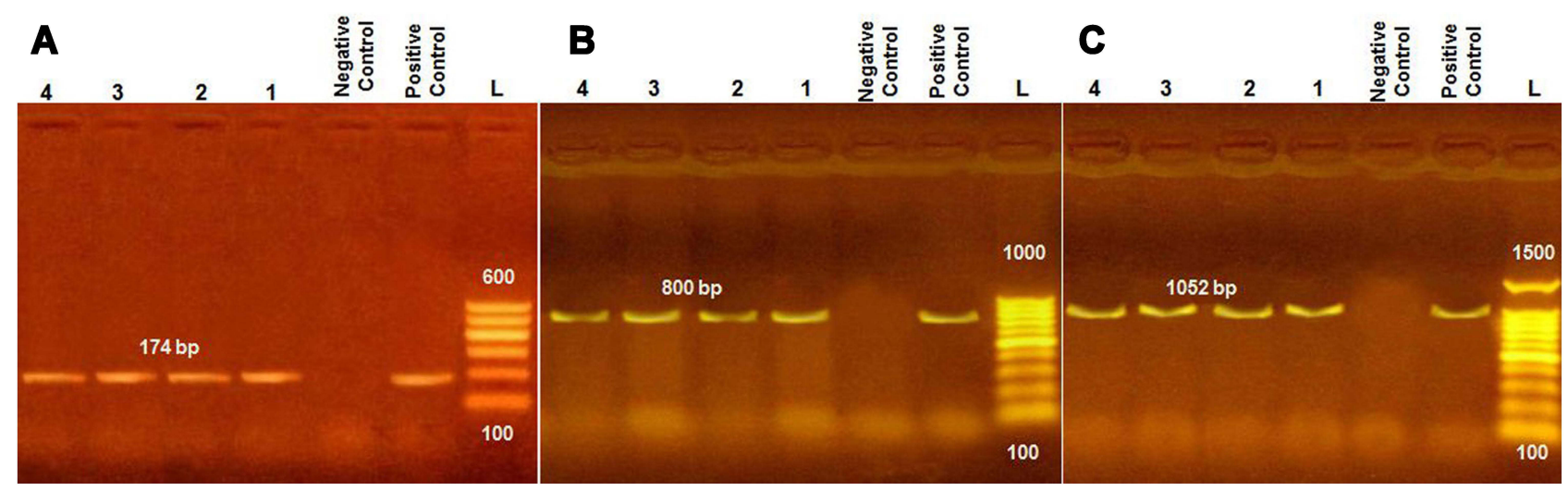

Figure I PCR products of amplified virulent genes identified in L. monocytogenes and visualized with agarose-gel electrophoresis. Molecular size of amplified DNA: I74 bp for hlyA (A), 800 bp for inlA (B), and 1052 bp for prfA (C). Lanes I-4, samples; L, 100 bp DNA ladder.

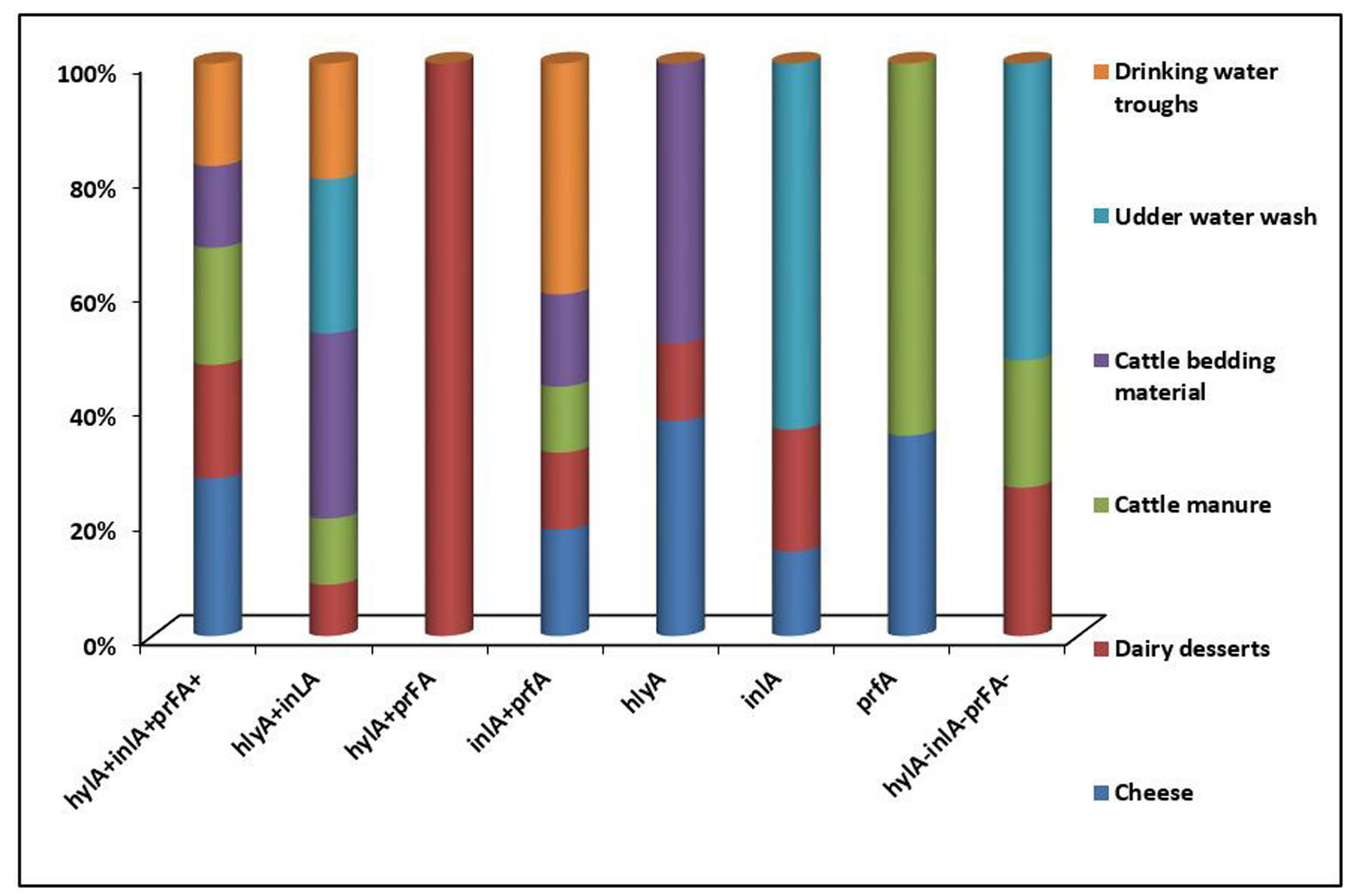

Figure 2 Distribution of $h l y A$, inlA, and prfA virulence genes in $L$. monocytogenes isolates $(n=50)$ recovered from cheese, dairy desserts, and environmental samples.

Log reductions for various disinfectants against susceptible and resistant $L$. monocytogenes clarified the marked lower efficacy of all the tested disinfectants against resistant strains. Increasing the contact time to 10 minutes did not improve the inhibitory power of disinfectants, eg, BioSentry 904 at its highest concentration 1:32 showed $\log$ reduction of 3.97 , which slightly improved to 4.23 after increasing the contact time to 10 minutes at the same concentration. The same phenomena were observed for BAC and Vanoquat disinfectants against resistant $L$. monocytogenes strains. In contrast, the most potent disinfectant against L. monocytogenes-susceptible/sensitive strains was Vanoquat, with $\log$ reductions of 6.64-8.42 $\log _{10}$, followed by BioSentry 904 with 6.47-8.14 $\log _{10}$, and the least powerful was BAC: 5.21-7.76 $\log _{10}$ (Figure 3 and Table S3). Screening of L. monocytogenes 

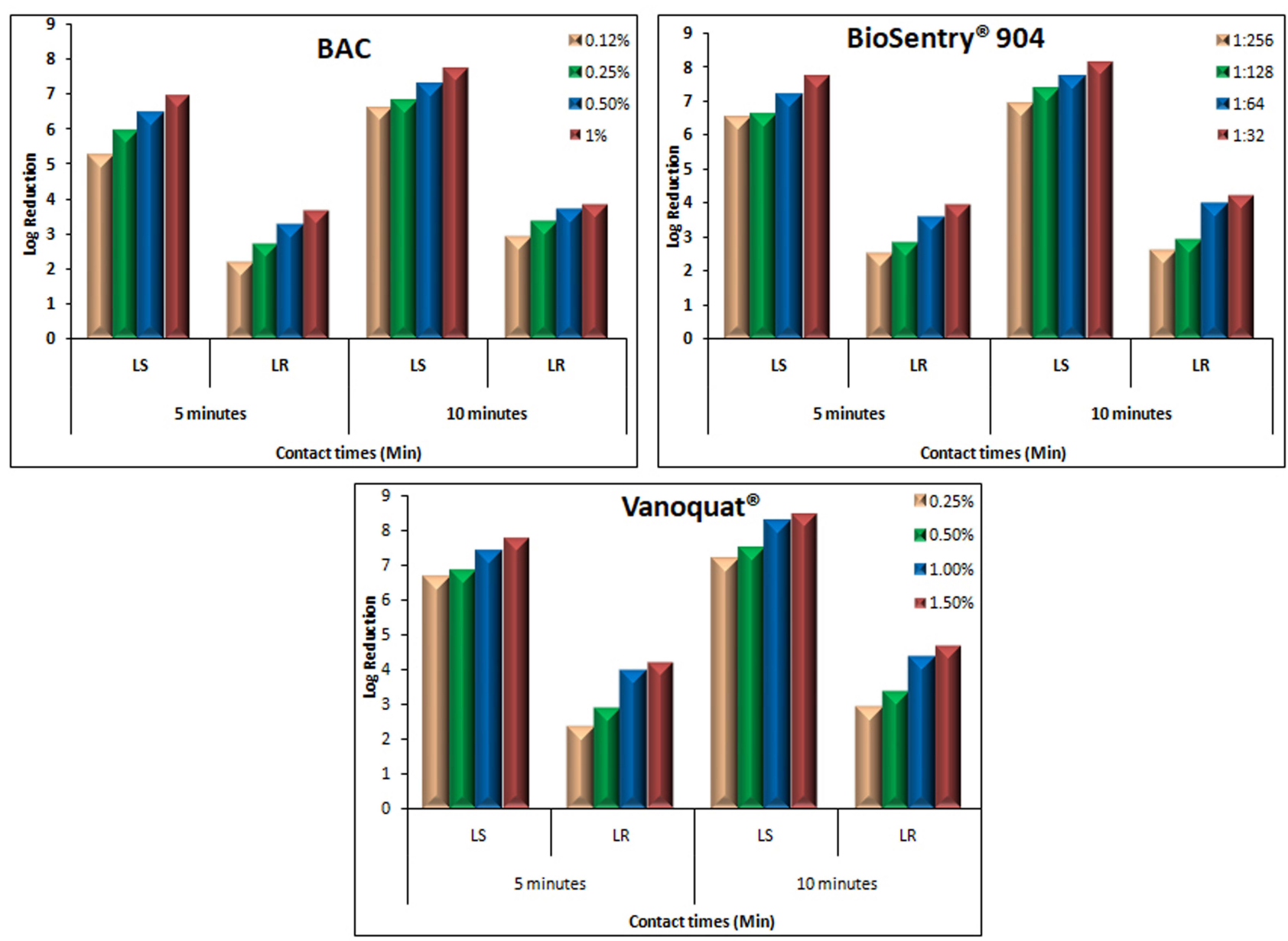

Figure 3 Log-reduction values of various QAC disinfectants (BAC, BioSentry 904, and Vanoquat) against L. monocytogenes-susceptible and -resistant strains isolated from dairy products using European suspension at various contact times.

strains for QAC-resistance genes revealed that 20 of 50 (40\%) carried $q a c H$ (Figure 4).

\section{Characterization of Ag-NPs-P. pavonica}

TEM showed the shape of Ag-NPs biosynthesized by $P$. pavonica extract (Figure 5). The image reveals that most NPs are spherical and some agglomerates in ellipsoidal form. Size, count, and aggregation of Ag-NPs were estimated by DLS. The total count of Ag-NPs was $5 \times 10^{5} \mathrm{NPs} /$ $\mathrm{mL}$ and average diameter $46.21 \mathrm{~nm}$. Ag-NPs synthesized by $P$. pavonica extract showed high negative -potential: $-25.4 \mathrm{mV}$. The polydispersity index (PDI) value of AgNPs was 0.638 and $Z$-average $142.6(\mathrm{~d} \bullet \mathrm{nm})$. The PDI value shows that the particles were polydispersed.

\section{Antibacterial Activity of Algal Extracts}

Acetone extracts of C. racemosa and J. rubens and petroleum ether extract of $P$. pavonica were potentially effective in inhibiting L. monocytogenes growth, with variable potency. The phytochemical constituents of those algal extracts were identified by GC-MS (Figure 7). The active compounds with retention time, molecular formula, molecular weight, and relative concentration (\%) for the algal extracts are presented in Table 5 and Table S4. The acetone extract of J. rubens and petroleum ether extract of P. pavonica were the most effective suppressors of $L$. monocytogenes at $3 \mathrm{mg} / \mathrm{mL}$, with inhibition zones of 7.4 and $9.2 \mathrm{~mm}$, respectively (Table 4). Moreover, the antibacterial activity of algal extracts and Ag-NPs- $P$. pavonica against $L$. monocytogenes was confirmed using liquid dilution (Figure 6A and B). When incubated with $L$. monocytogenes for 24 hours, all tested materials exhibited strong bactericidal activity in a dose-dependent manner. The petroleum ether extract of $P$. pavonica was very effective against $L$. monocytogenes, resulting in severe reduction in bacteria CFU (13 $\log _{10}$ orders of killing) at $3 \mathrm{mg} / \mathrm{mL}$; however, the acetone extracts of $J$. rubens and C. racemosa showed much less $\log$ reduction (8 $\log _{10}$ orders of killing) of $L$. 


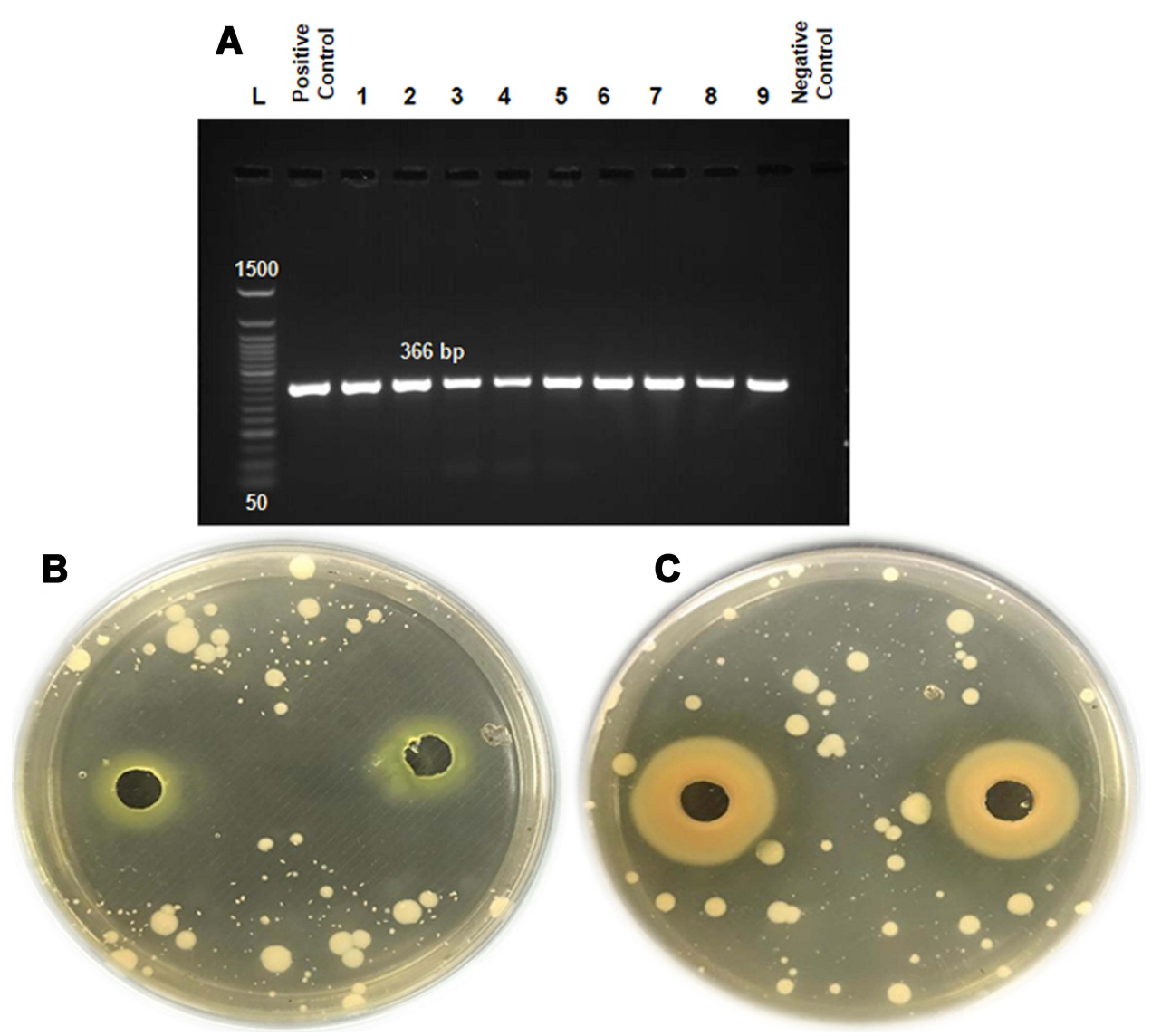

Figure 4 (A) The qacH gene in L. monocytogenes-tolerant strains conferred resistance to QAC disinfectants. (B, C) Agar-well diffusion assay showed inhibition zones for susceptible and resistant $L$. monocytogenes strains.
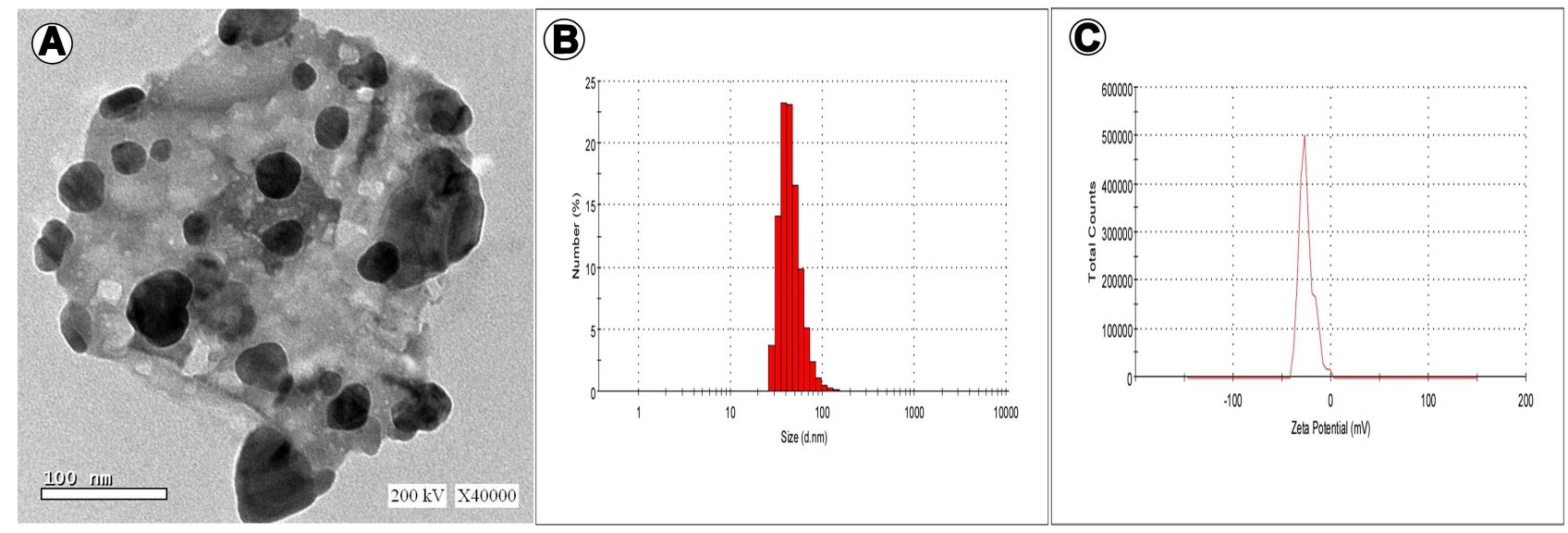

Figure 5 TEM (A), size distribution (B), and -potential (C) of Ag-NPs biosynthesized by Padina pavonica.zzzz

monocytogenes at the same concentration of $3 \mathrm{mg} / \mathrm{mL}$ (Figure 6A). Also, Ag-NPs-P. pavonica exhibited effective bactericidal action (7-8 $\log _{10}$ orders of killing) at concentration of $1.6 \times 10^{5}$ NPs-mL (Figure $6 \mathrm{~B}$ ).

The results of GC-MS analysis showed 10, 23, and 24 compounds in the acetone extracts of C. racemosa and $J$. rubens and petroleum ether extract of $P$. pavonica, respectively. The most prevalent compounds in C. racemosa acetone extract were 9-octadecenoic acid (Z)-, methyl ester (48.31\%), $\beta$-sitosterol (16.23\%), and hexadecanoic acid methyl ester (3.94\%), while those of $J$. rubens acetone extract were 11-octadecenoic acid, methyl ester 
Table 4 Antibacterial-screening tests of algal extracts $(1.5$ and $3 \mathrm{mg} / \mathrm{mL}$ ) and disinfectants (various concentrations*) against $L$. monocytogenes determined by zone of inhibition using agar-well diffusion ${ }^{\mathrm{a}, \mathrm{b}}$

\begin{tabular}{|c|c|c|c|c|}
\hline \multirow[t]{2}{*}{ (A) Algae } & \multirow[t]{2}{*}{ Extract } & \multicolumn{3}{|c|}{ Inhibition-zone diameter $(\mathrm{mm})$} \\
\hline & & \multicolumn{2}{|c|}{$1.5 \mathrm{mg} / \mathrm{mL}$} & $3 \mathrm{mg} / \mathrm{mL}$ \\
\hline Caulerpa racemosa & \multicolumn{2}{|l|}{ Acetone } & $3.1 \pm 0.44$ & $6.5 \pm 0.35$ \\
\hline Jania rubens & \multicolumn{2}{|l|}{ Acetone } & $5.8 \pm 0.03$ & $7.4 \pm 0.15$ \\
\hline Padina pavonica & \multirow[t]{2}{*}{ Petroleum ether } & & $6.3 \pm 0.41$ & $9.2 \pm 0.47$ \\
\hline DMSO & & & No inhibition & No inhibition \\
\hline \multirow[t]{2}{*}{ (B) Disinfectant* } & \multicolumn{4}{|c|}{ Inhibition-zone diameter $(\mathrm{mm})$} \\
\hline & \multicolumn{4}{|c|}{ Various concentrations* } \\
\hline BAC & $18.3 \pm 0.88$ & $19.3 \pm 0.88$ & $2.7 \pm 0.33$ & $27.0 \pm 0.58$ \\
\hline BioSentry 904 & $21.2 \pm 0.38$ & $22.5 \pm 0.29$ & $27.0 \pm 0.12$ & $30.6 \pm 0.35$ \\
\hline Vanoquat & $28.0 \pm 0.58$ & $32.6 \pm 0.34$ & $37.3 \pm 0.88$ & $40.0 \pm 0.15$ \\
\hline
\end{tabular}

Notes: ${ }^{a}$ Means \pm SE of three replicates. Diameter of well $(7 \mathrm{~mm})$ not included. ${ }^{b}$ Only positive values of the examined algae extracts were recorded. ${ }^{*} \mathrm{BAC} 0.12 \%, 0.25 \%, 0.5 \%$, I\%; BioSentry 904 I:32, I:64, I: I28, I:256; Vanoquat $0.25 \%, 0.25 \%, 0.5 \%, 1.5 \%$ ).

Table 5 Major bioactive chemical compounds identified in the most effective algal extracts

\begin{tabular}{|c|c|c|c|c|c|c|}
\hline Algae & Extract & $\begin{array}{l}\text { Retention time } \\
\text { (minutes) }\end{array}$ & Compound name & $\begin{array}{l}\text { Molecular } \\
\text { formula }\end{array}$ & $\begin{array}{c}\text { Molecular } \\
\text { weight }\end{array}$ & $\begin{array}{c}\text { Peak } \\
\text { area (\%) }\end{array}$ \\
\hline $\begin{array}{l}\text { Caulerpa } \\
\text { racemosa }\end{array}$ & Acetone & $\begin{array}{l}32.29 \\
36.95 \\
41.28 \\
45.23 \\
61.17\end{array}$ & $\begin{array}{l}\text { Hexadecanoic acid, methyl ester } \\
\text { 9-Octadecenoic acid (Z)-, methyl ester } \\
\text { Heptacosanoic acid, methyl ester } \\
\text { Docosanoic acid, methyl ester } \\
\beta \text {-Sitosterol }\end{array}$ & $\begin{array}{l}\mathrm{C}_{17} \mathrm{H}_{34} \mathrm{O}_{2} \\
\mathrm{C}_{19} \mathrm{H}_{36} \mathrm{O}_{2} \\
\mathrm{C}_{28} \mathrm{H}_{56} \mathrm{O}_{2} \\
\mathrm{C}_{23} \mathrm{H}_{46} \mathrm{O}_{2} \\
\mathrm{C}_{29} \mathrm{H}_{50} \mathrm{O}\end{array}$ & $\begin{array}{l}270 \\
296 \\
424 \\
354 \\
414\end{array}$ & $\begin{array}{c}3.94 \\
48.31 \\
2.8 \\
2.08 \\
16.23\end{array}$ \\
\hline Jania rubens & Acetone & $\begin{array}{l}3.46 \\
6.36 \\
15.12 \\
17.67 \\
18.38 \\
20.36 \\
21.09\end{array}$ & $\begin{array}{l}\text { I3-Tetradecynoic acid, methyl ester } \\
\text { I7-Octadecynoic acid } \\
\text { Tetradecanoic acid, methyl ester } \\
\text { Hexadecanoic acid, methyl ester } \\
\text { Hexadecanoic acid, ethyl ester } \\
\text { II-Octadecenoic acid, methyl ester } \\
\text { Ethyl Oleate }\end{array}$ & $\begin{array}{l}\mathrm{C}_{15} \mathrm{H}_{26} \mathrm{O}_{2} \\
\mathrm{C}_{18} \mathrm{H}_{32} \mathrm{O}_{2} \\
\mathrm{C}_{15} \mathrm{H}_{30} \mathrm{O}_{2} \\
\mathrm{C}_{17} \mathrm{H}_{34} \mathrm{O}_{2} \\
\mathrm{C}_{18} \mathrm{H}_{36} \mathrm{O}_{2} \\
\mathrm{C}_{19} \mathrm{H}_{36} \mathrm{O}_{2} \\
\mathrm{C}_{20} \mathrm{H}_{38} \mathrm{O}_{2}\end{array}$ & $\begin{array}{l}238 \\
280 \\
242 \\
270 \\
284 \\
296 \\
310\end{array}$ & $\begin{array}{c}4.34 \\
3.26 \\
3.23 \\
26.54 \\
2.37 \\
38.51 \\
2.55\end{array}$ \\
\hline $\begin{array}{l}\text { Padina } \\
\text { pavonica }\end{array}$ & $\begin{array}{l}\text { Petroleum } \\
\text { ether }\end{array}$ & $\begin{array}{l}18.90 \\
22.22 \\
22.47 \\
26.34 \\
28.10 \\
29.03\end{array}$ & $\begin{array}{c}\text { n-Hexadecanoic acid } \\
\text { Ethanone, I-(7,8-dihydro-3-hydroxy-4- } \\
\text { propyl-2-naphthalenyl)- } \\
\text { Erucic acid } \\
\text { Octacosane } \\
\text { I5-Nonacosanone } \\
\text { I-Heptatriacotanol }\end{array}$ & $\begin{array}{l}\mathrm{C}_{16} \mathrm{H}_{32} \mathrm{O}_{2} \\
\mathrm{C}_{15} \mathrm{H}_{18} \mathrm{O}_{2} \\
\mathrm{C}_{22} \mathrm{H}_{42} \mathrm{O}_{2} \\
\mathrm{C}_{28} \mathrm{H}_{58} \\
\mathrm{C}_{29} \mathrm{H}_{58} \mathrm{O} \\
\mathrm{C}_{37} \mathrm{H}_{76} \mathrm{O}\end{array}$ & $\begin{array}{l}256 \\
230 \\
338 \\
394 \\
422 \\
536\end{array}$ & $\begin{array}{c}3.95 \\
1.99 \\
7.47 \\
38.41 \\
33.05 \\
7.51\end{array}$ \\
\hline
\end{tabular}

(38.51\%), hexadecanoic acid methyl ester (26.54\%), 13tetradecynoic acid methyl ester (4.34\%), 17-octadecynoic acid (3.26\%), and tetradecanoic acid methyl ester (3.23\%). The most abundant compounds in the P. pavonica petroleum ether extract were octacosane $(38.41 \%)$, 15nonacosanone (33.05\%), 1-heptatriacotanol (7.51\%), erucic acid (7.47\%), and $n$-hexadecanoic acid $(3.95 \%$; Table 5, Table S4, and Figure 7). 
A (A1) Antibacterial activity of algae extract against $L$.

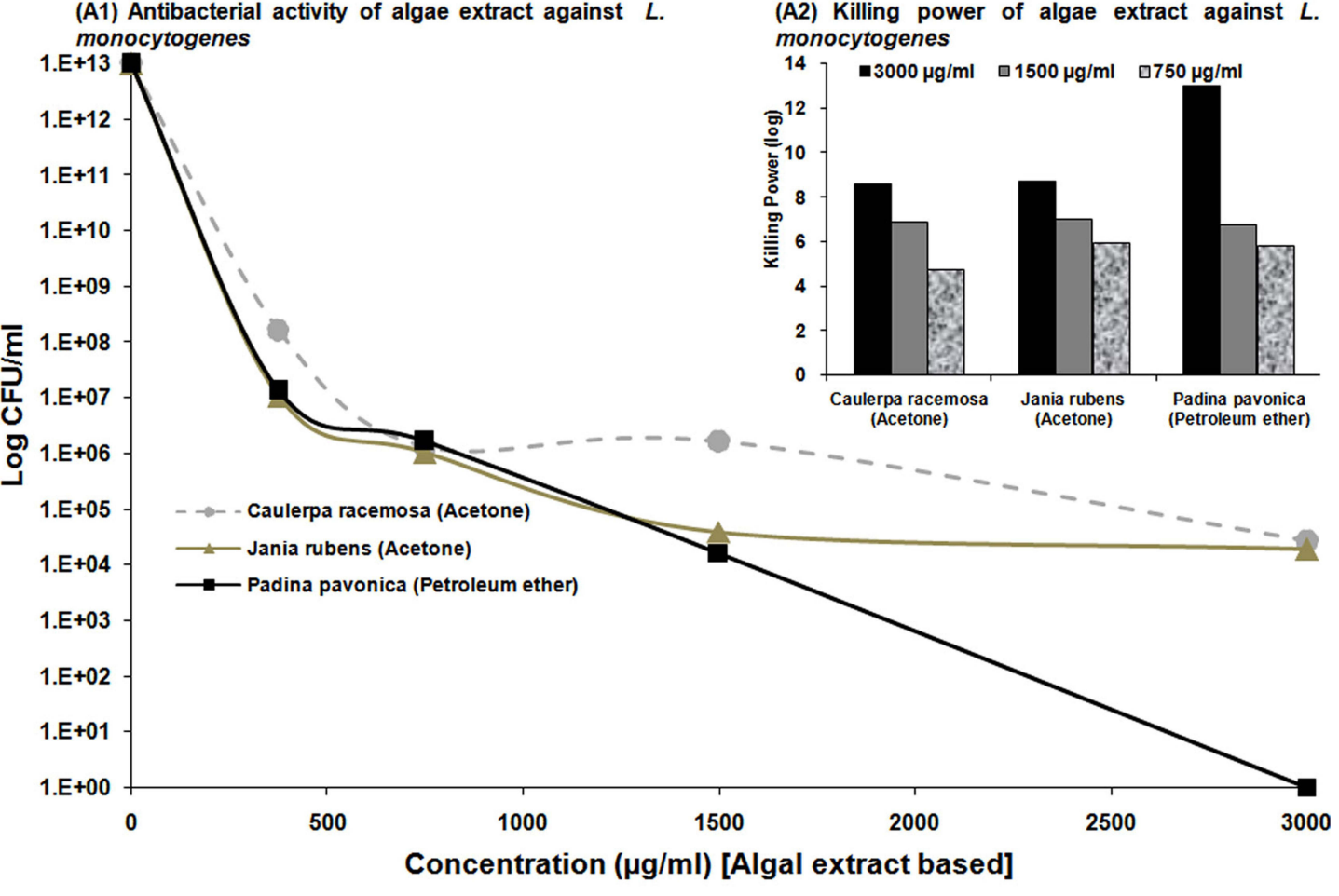

B

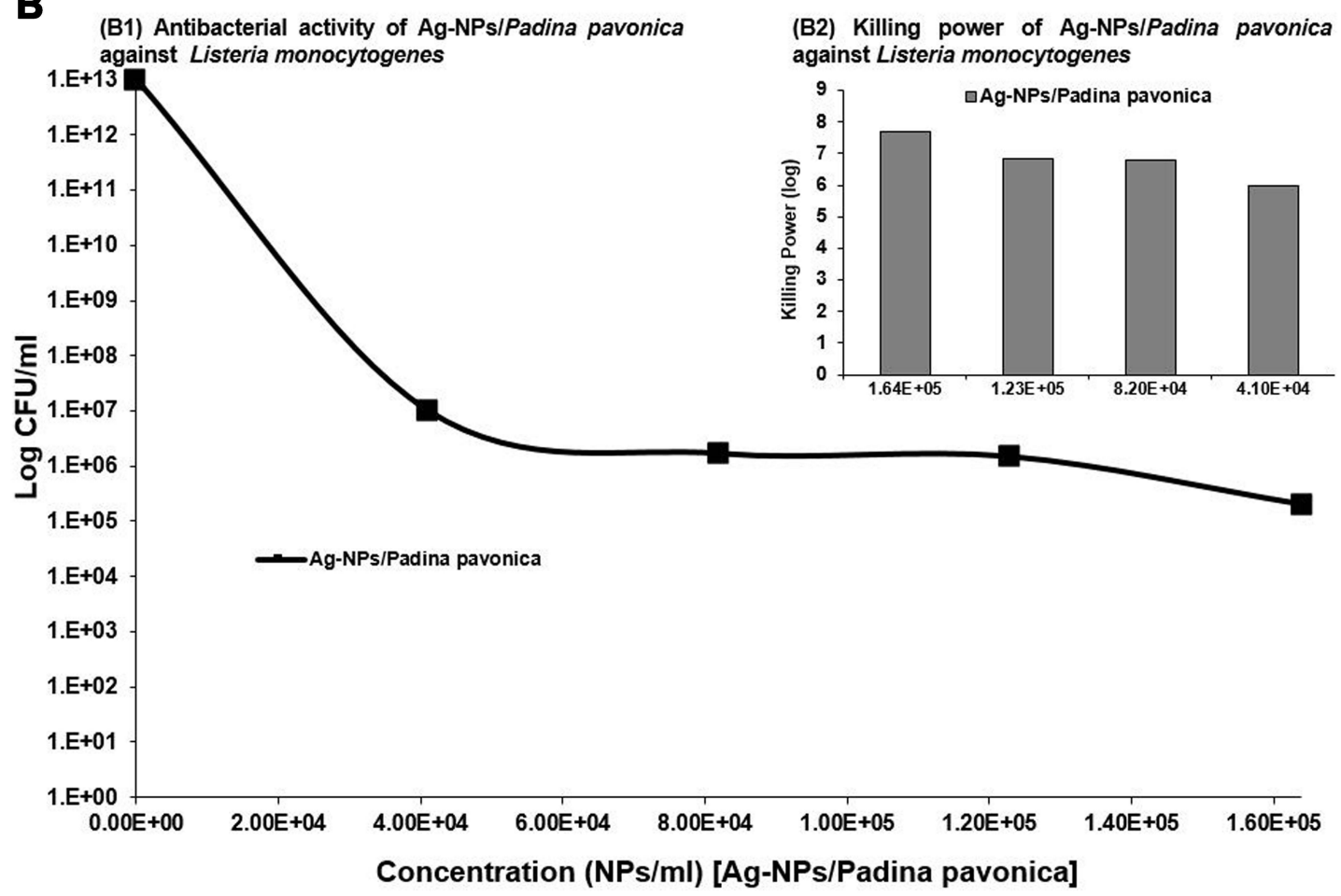

Figure 6 Antibacterial activity and killing power of algae extracts (A) and $1.64 \times 10^{5}, 1.23 \times 10^{5}, 8.2 \times 10^{4}$, and $4.10 \times 10^{4} \mathrm{Ag}$-NPs-Padina pavonica (B) against L. monocytogenes at concentrations of 750, 1,500, and 3,000 $\mathrm{\mu g} / \mathrm{mL}$. Data presented as log CFU/mL. Only positive values of the examined algal extracts and NPs were recorded. Assays were performed in triplicate. 

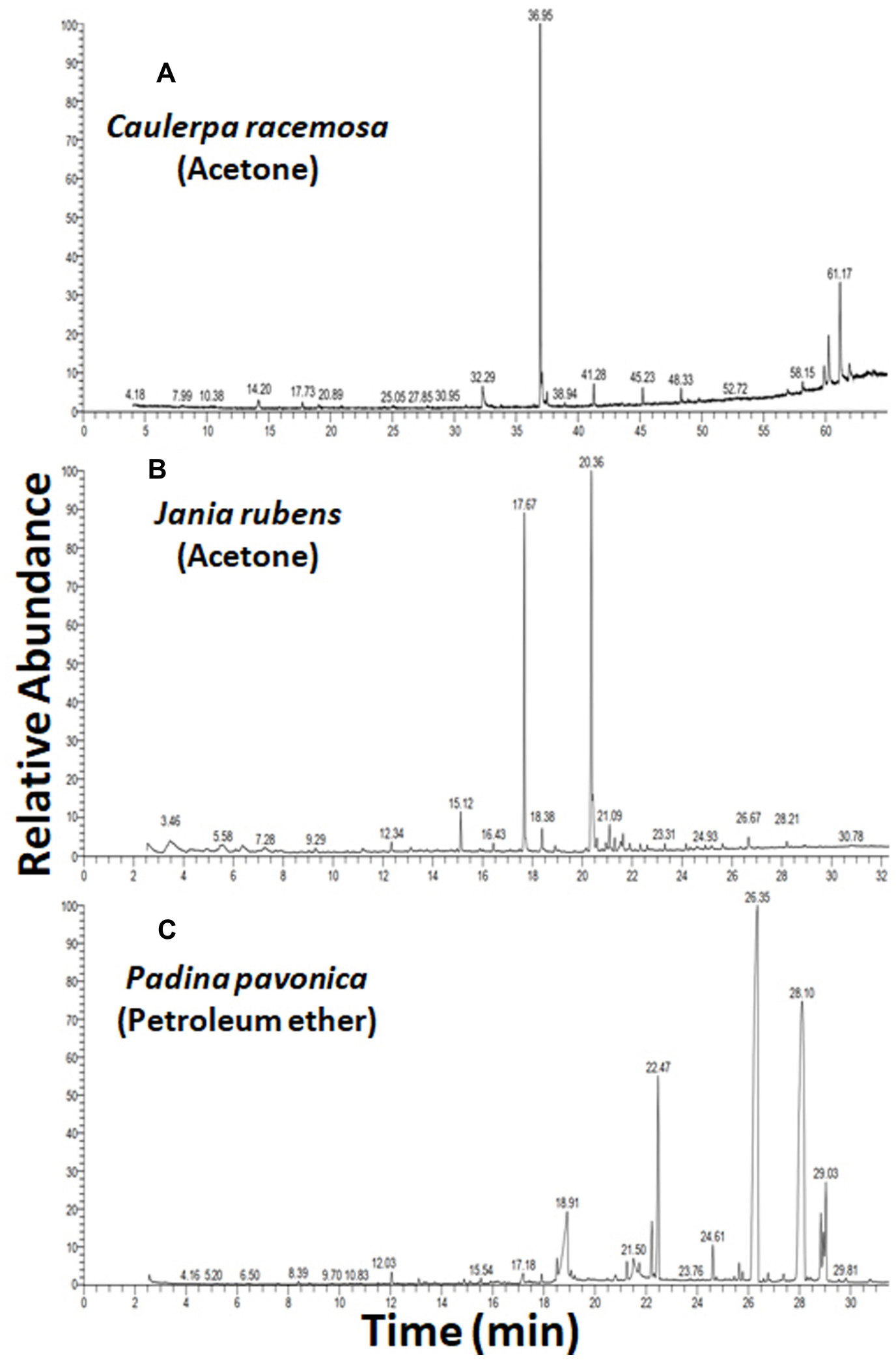

Figure 7 GC-MS of the most effective algal extracts. 


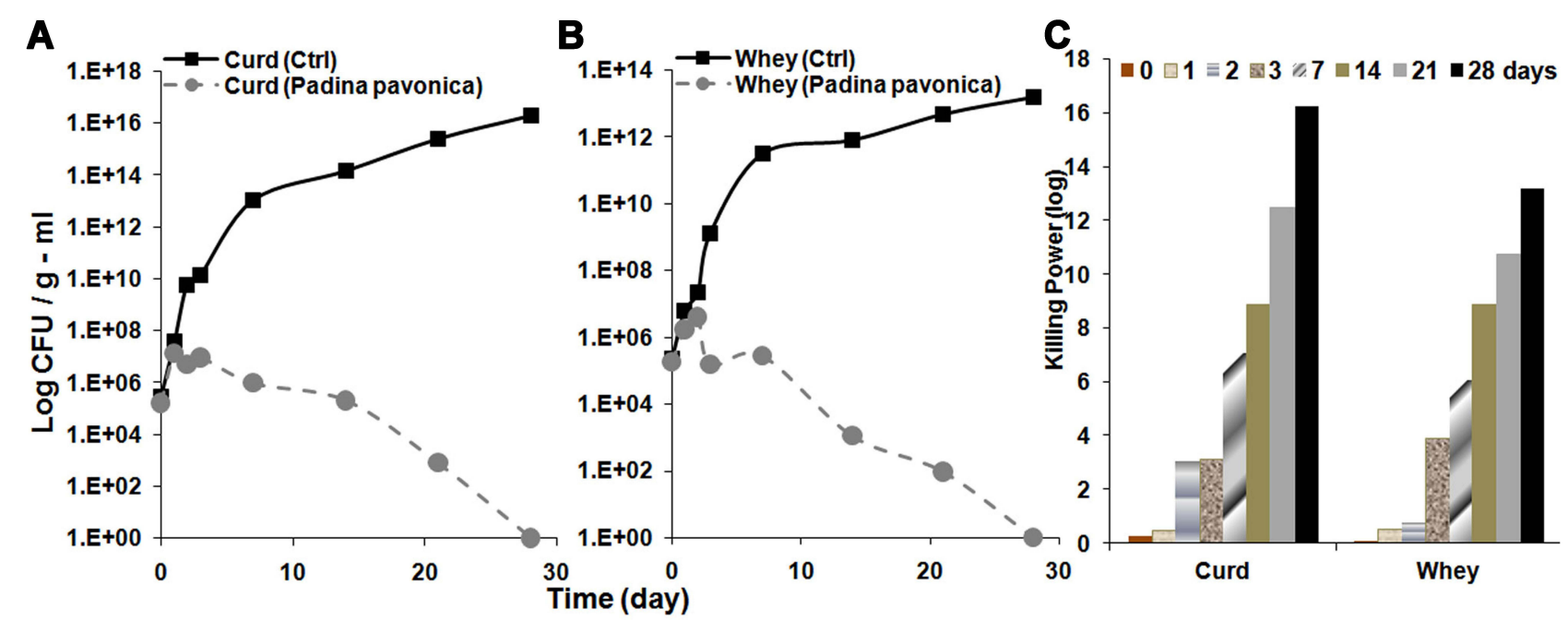

Figure 8 Effect of Padina pavonica extract $(750 \mu g / \mathrm{mL})$ on the growth of L. monocytogenes in experimentally manufactured ripened white cheese: (A) cheese curd and (B) cheese whey. (C) Killing power of Padina pavonica extract $(750 \mu \mathrm{g} / \mathrm{mL})$ against L. monocytogenes in comparison with time (days) in both curd and whey of experimentally manufactured ripened white cheese.

\section{Impact of $P$. pavonica Extract on Growth and Survival of Virulent L. monocytogenes in Cheese and Whey}

This part of the study was designed based on the results exhibited by $P$. pavonica in the in vitro trials for antimicrobial activity against $L$. monocytogenes. An isolate of L. monocytogenes with toxin-virulence profile 1 (Table s2) and phenotypically and genotypically resistant to QACs had been inoculated in raw milk. The behaviour of $L$. monocytogenes during cheese manufacturing and storage in both the presence and absence of the P. pavonica extract is illustrated in Figure 8. Initial numbers of L. monocytogenes in control cheese curd and whey were 5.46 and $5.36 \log \mathrm{CFU} / \mathrm{g}$, respectively, while in P. pavonica extract cheese curd and whey were 5.2 and $5.2 \log \mathrm{CFU} / \mathrm{mL}$, respectively. In control cheese curd and whey, L. monocytogenes grew well, reaching 10, 14, and $16 \log \mathrm{CFU} / \mathrm{g}$ in cheese curd and 9, 11, 13 $\log \mathrm{CFU} / \mathrm{mL}$ in cheese whey after 3, 14, and 28 days, respectively, under refrigeration. On the other hand, $L$. monocytogenes loads were reduced at different levels for curd and whey where the cheese had been treated with $P$. pavonica extract. After 3 and 14 days of ripening, L. monocytogenes counts in cheese curd were reduced by more than 4 and $9 \log \mathrm{CFU} / \mathrm{g}$; while in cheese whey counts were reduced by more than 4 and $8 \log \mathrm{CFU} / \mathrm{mL}$ compared with the cheese inoculated with $L$. monocytogenes without $P$. pavonica extract. Pathogen levels continued to decline during storage, and finally at the 28th day of storage in either cheese curd or whey L. monocytogenes could not be detected, indicating the bactericidal power of P. pavonica extract against $L$. monocytogenes in cheese curd and whey.

\section{Discussion}

Rice milk and kareish cheese had the highest isolation rate of $L$. monocytogenes, followed by feta cheese and custard. The high prevalence of $L$. monocytogenes in these dairy products could be due to environmental pollution or unsanitary production methods and food storage. However, it could also be due to the use of unpasteurized milk. Also, Listeria spp. can continue to grow in the refrigerator and low salt-concentration environments. Moreover, cheese is the perfect environment for the growth of microorganisms. ${ }^{36}$ On the other hand, no processed cheese was found to be contaminated with Listeria spp., emphasizing the fact that processed cheese is considered and classified as one that does not support the growth of L. monocytogenes under reasonable foreseeable conditions of distribution and storage. ${ }^{37}$ However, postprocessing contamination of the product should be strictly avoided, as the pathogen can survive in the product for extended periods, particularly under refrigeration $\left(4^{\circ} \mathrm{C}\right)$.

In animals, listeriosis is mainly a disease of ruminants. ${ }^{38}$ Animals are commonly asymptomatic intestinal carriers, frequently shedding the organism and maintaining its populations in the environment. Especially, bovine hosts may amplify ingested L. monocytogenes and thus serve as a critical factor in maintaining high prevalence of the pathogen 
on cattle farms. ${ }^{39}$ Farm-specific factors, such as using untreated livestock manure as fertilizer, contamination of irrigation water through runoff from livestock facilities, ${ }^{40}$ large group sizes, unhygienic practices during milking, and cattle drinking from a trough, might greatly affect the prevalence of L. monocytogenes. Good farm-level practices can thus be utilized to reduce the prevalence of this pathogen on the farm and possibly further in the food chain. ${ }^{41}$

In the present study, virulent $L$. monocytogenes strains harboring $h l y A$, inlA, and $\operatorname{prf} A$ were detected in 21 of 31 $(67.7 \%)$ and 14 of $19(73.7 \%)$ L. monocytogenes isolates obtained from dairy products and environmental samples, respectively (Table 3 ). This finding was less than previous studies in which virulence genes were detected in L. monocytogenes isolated from dairy products. ${ }^{42,43}$ More than half the L. monocytogenes isolated from dairy products $(61.3 \%)$ and environmental samples (68.4\%) was positive for more than one virulence-associated gene, while none of 31 (29\%) and 5 of $19(26.3 \%)$ from dairy products and environmental samples, respectively, harbored only one virulence-associated gene (Figure 2 and Table S2) and were likely less virulent than those with multiple virulence-associated genes.

The current study revealed QAC disinfectants exhibited clear inhibition zones with complete lack of growth for some L. monocytogenes strains, while others showed tolerance against the QACs and had the ability to survive with complete growth inside the inhibition zones (Table 4 and Figure 4). All strains were further checked using the European suspension test to clarify the killing power/log reduction of the various disinfectants. This revealed a marked difference in killing power/log reductions of all disinfectants between susceptible L. monocytogenes strains and resistant. To confirm the findings, we used the QAC disinfectants at various concentrations and variable contact times against both a single sensitive strain and another single resistant strain of $L$. monocytogenes selected from those isolated from dairy products. Even after exposure of the resistant L. monocytogenes strains to higher concentrations and prolonged contact times, the log reduction was still very low for resistant strains compared to susceptible ones (Table S3 and Figure 3).

Our data revealed that BAC-QAC disinfectants had good bactericidal activity against L. monocytogenes-susceptible strains at all the tested concentrations for both contact times. However, against resistant strains, log reductions were much lower at all concentrations for both contact times. The association of the lower log reductions with resistant L. monocytogenes strains was observed among all the tested QAC disinfectants, including BioSentry 904 and Vanoquat (Table S3 and Figure 3).

Resistance to QACs disinfectants, such as BAC, is especially relevant to Listeria's adaptations in food-related environments, as these compounds are used extensively in food processing, in retail, and for household or personal use. ${ }^{44}$ BAC-resistant strains of $L$. monocytogenes have been implicated in multistate outbreaks of listeriosis, and have frequently been isolated from food-processing plants. However, the genetic basis for BAC resistance in L. monocytogenes remains poorly understood. The tolerance of $L$. monocytogenes to BAC varies between strains. ${ }^{45}$ Resistance to BAC has been observed in different countries. ${ }^{8}$ BAC resistance of L. monocytogenes isolated from food and the processing-plant environment has been found to range from $10 \%{ }^{7}$ to as much as $42 \%-46 \%$.

Different genetic markers have been identified that confer L. monocytogenes resistance to QACs, including the qacH gene of transposon $\operatorname{Tn} 6188 .^{8}$ The presence and distribution of these genes have been anticipated to have a role in the survival and growth of $L$. monocytogenes in food-processing environments where QAC-based disinfectants are in common use. Some studies have shown that $L$. monocytogenes harbouring the QAC-resistance gene qacH are prevalent in the food industry and that residuals of QAC may be present after sanitation that result in a growth advantage for bacteria with such resistance genes. ${ }^{47}$

All strains were screened for the presence of $q a \mathrm{cH}$. Resistance to QACs was associated with $q a c H$ in $40 \%$ of the tested strains, while the remainder $(60 \%)$ did not harbor that genetic determinant and were classified as sensitive to QACs (Figure 4). Previous research has shown that resistance to BAC was associated with $q a c H$ in a majority $(80 \%)$ of the tested strains. ${ }^{48}$ Furthermore, qacH has been found in $22 \%$ of $L$. monocytogenes isolates. ${ }^{47}$ L. monocytogenes strains harbor the transposon Tn6188, responsible for increased tolerance against QAC through qacH, and it has been suggested that this contributes to survival and persistence. ${ }^{49}$

The persistent strains of $L$. monocytogenes in food-processing environments after cleaning and disinfection contribute to many factors, the most important being the presence of organic material. ${ }^{50}$ Inadequate disinfection may produce resistance to the disinfectant as a result of selection or adaptation through regular exposure to sublethal concentrations. ${ }^{7}$ In dairy/foodprocessing environments, L. monocytogenes is exposed to different disinfectants and sanitizers, sometimes at subinhibitory concentrations. This is particularly true for disinfectants that are not fully biodegradable and may persist in sewage for long periods. QACs are considered to have poor 
biodegradability, so the contact between bacteria and QACs may be prolonged and consequently cause frequent exposure of microbial communities to subinhibitory concentrations of QACs, facilitating the development of resistance of certain strains over time. ${ }^{51,52}$

It has become increasingly difficult to protect human health from the adverse effects of $L$. monocytogenes pathogens. Limited use of chemical preservatives, susceptibility, toxicity, microbial resistance, and adverse effects on human health increase the need for pure, healthier, safer, and potentially successful antibacterial agents. Therefore, the antibacterial activity of algal extracts can provide key materials to be used as natural preservatives to ensure healthy and safe food. Potent antibacterial activity was observed for P. pavonica in petroleum ether extract against L. monocytogenes, resulting in a severe reduction in the CFU of bacterial load (13 $\log _{10}$ orders of killing) at $3 \mathrm{mg} / \mathrm{mL}$. However, $3 \mathrm{mg} / \mathrm{mL}$ acetone extract of C. racemosa and J. rubens showed much less log reduction (8 $\log _{10}$ orders of killing) of L. monocytogenes.

Various studies have evaluated the antimicrobial activity of marine seaweed, ie, P. pavonica, C. racemosa, and J. rubens. The solvents petroleum ether, acetone, chloroform, methanol, ethanol, hexane, ethyl acetate, and water have been used for algal extraction to explore antibacterial activity against both Gram-positive and Gram-negative bacteria, including $L$. monocytogenes. $^{31}$ Globally, P. pavonica ethanolic extract exhibits the highest activity against $L$. monocytogenes growth, with an inhibition zone of $14 \mathrm{~mm}^{53}$ and $13 \mathrm{~mm}$ against other Listeria spp. ${ }^{54}$ In addition, methanolic ethanol, chloroform, acetone, ethyl acetate, and hexane extracts of $P$. pavonica show have shown activity against the growth of $L$. monocytogenes with $16 \pm 0.27,14 \pm 0.11,13 \pm 0.19,11 \pm 0.22,15 \pm 0.27$, and $9 \pm 0.09 \mathrm{~mm}$ inhibition zones, respectively. ${ }^{55}$ Caulerpa spp. exhibit antibacterial activity against the growth of L. monocytogenes, with a $20.6 \pm 0.6 \mathrm{~mm}$ inhibition zone. ${ }^{56}$ Moreover, it has been reported that $C$. racemosa extract exhibits significant antimicrobial activity against pathogenic bacteria in human food. ${ }^{57}$ The great efficacy of marine seaweed extracts against L. monocytogenes could be due to active phytochemicals and metabolite compounds, in addition to fatty acids and their derivatives. $^{13}$

The potent antibacterial effects of $P$. pavonica petroleum ether extract against L. monocytogenes could be attributed to the most abundant detected phytochemical bioactive compounds: octacosane (38.41\%), 15-nonacosanone $(33.05 \%)$, 1-heptatriacotanol $(7.51 \%)$, erucic acid (7.47\%), and $n$-hexadecanoic acid (3.95\%).
In vitro data presented in previous literature has proved that octacosane and 15-nonacosanone have potent antimicrobial activity against a wide range of bacterial species, including L. monocytogenes, show a nontoxic, nonmutagenic, and nontumorigenic properties, and could be potential antibacterial drugs. Moreover, octacosane and 15nonacosanone show such antimicrobial properties as antibacterial, antifungal, and antiviral activity, which can be used to prevent microbial growth in wide-ranging applications such as food, cosmetics, and drug molecules. ${ }^{58}$

It could be concluded that those molecules identified have potential antimicrobial activities, and notably that the extracts of the algae where they are abundant also show potent antimicrobial activities. These algal species could be more effective against bacterial infection than traditional bactericidal agents. Therefore, natural preservatives can be considered to provide healthy, safe food via their stable biologically active compounds without the unpleasant effects of chemical ones. Also, this study provides insights into designing novel antibacterial agents for food preservation or clinical use.

Ripened soft white cheese is the most popular dairy product in Egypt and other Middle Eastern countries, and because of its popularity, upon contamination it can be a major cause of listeriosis, as L. monocytogenes has been reported to be the most common foodborne pathogen associated with cheese ${ }^{7,59}$ and cheese is a potential reservoir for L. monocytogenes due to higher nutrient quality and greater contamination risk because increased handling. In Egypt, the incidence of L. monocytogenes has been evaluated in locally produced dairy products, such as cheese, and its higher incidence in dairy products, especially cheese, may be attributed to traditional unhygienic manufacturing techniques, resulting in linkage of these products to several outbreaks of listeriosis. ${ }^{42,60}$ These results indicate the need for control strategies to prevent the dispersion of L. monocytogenes through cheese and the public-health hazards linked to consumption of these products. El Shinaway et $\mathrm{al}^{61}$ described the ability of L. monocytogenes to survive and resist acidic, refrigeration, or freezing conditions during manufacturing, distribution, and storage of dairy products. We cannot rely solely upon these processes to control $L$. monocytogenes to provide safe products for human consumption. Green, brown, and red algae with antioxidant activity can be safely consumed by humans. ${ }^{62,63}$

The present research enabled us to discover the ability of algal species as antibacterial agents against $L$. monocytogenes and their ability to control L. monocytogenes in artificially inoculated white cheese, thereby offering strong reasons for the development of antimicrobial formulations for food 
preservation. The data clarified that in the absence of $P$. pavonica extract, L. monocytogenes grew well, reaching 16 $\log \mathrm{CFU} / \mathrm{g}$ after 28 days under refrigeration. The excessive growth of L. monocytogenes in the control cheese is consistent with previous studies illustrating high growth potential of bacterial pathogens in white cheeses. ${ }^{64}$

Our results showed that the $P$. pavonica extract reduced the population of $L$. monocytogenes in white cheese by more than 4 and $9 \log \mathrm{CFU} / \mathrm{g}$ after 3 and 14 days of storage, respectively, following the addition of the algal extract, and finally at the 28th day of storage, L. monocytogenes could not be detected. There have been few previous studies in relation to algal extracts incorporated into model food systems to challenge their antimicrobial efficacy against food microorganisms, especially L. monocytogenes. Cox et $\mathrm{al}^{12}$ reported antimicrobial activity of Himanthalia elongata extract at varying concentrations $(1 \%, 5 \%$, and $10 \%)$ against $L$. monocytogenes in protein and carbohydrate model food systems. The extract provided complete bacterial inhibition, with bactericidal and bacteriostatic effects in carbohydrate and protein model food systems, respectively. Such extracts may have a multipurpose functionality, including antimicrobial and antioxidant bioactivity, which could potentially increase shelf life, safety, and quality of a wide range of food products.

Cho et $\mathrm{al}^{65}$ investigated making a functional drinkable milk product using algal extract powder (Chlorella spp.), with evaluation of quality maintenance. They demonstrated that preservation of the dairy products with the addition of Chlorella extract was relatively good at $4^{\circ} \mathrm{C}$ for 15 days. The results of sensory evaluation indicated that color and taste were significantly acceptable. In the current study, the addition of $P$. pavonica extract to cheese not only increased its microbiological safety but also increased the quality and smoothness of the cheese-curd texture, and the $\mathrm{pH}$ of the cheese by the end of the experiment was 4.32. Our results are in accordance with Jeon, ${ }^{66}$ who investigated the effect of addition of Chlorella algal extract on the microbial and sensory quality of processed cheese. Their results suggested that processed cheese with improved microbial quality can be made by the addition of microalgae, due to its ability to inhibit microorganisms. Moreover, processed cheese prepared with Chlorella was higher on descriptive analysis scores for color, mouth feel, hardness, and springiness.

\section{Conclusion}

In the current study, the petroleum ether extract of the seaweed tested showed strong antimicrobial efficacy against L. monocytogenes in cheese. At a concentration of $3 \mathrm{mg} / \mathrm{mL}$, L. monocytogenes was remarkably inhibited upon exposure to $P$. pavonica in petroleum ether extract, resulting in $13 \log _{10}$ orders of killing. QAC disinfectant (alkyl [ $\left.\mathrm{c}_{12-16}\right]$ dimethyl BAC) at a concentration of $1.5 \%$ for 5 minutes' contact resulted in a $7.7 \mathrm{log}$ reduction. However, efficacy was improved to $8.4 \log$ reduction through increasing the contact time to 10 minutes at the same concentration. On the in vitro level, the study provides promising findings for using the algal extract as an antimicrobial agent in food or drink products. Further investigations for the identification of promising algal species, standardization of analytical methods, isolation of compounds through bioassay-guided fractionation, detailed chemical characterization and evaluation of their safety, evaluation of synergistic effects among the components, and efforts to enhance yields and lower extraction costs are needed. Detection of disinfectant-resistance genes will help us in understanding the mechanisms of resistance and to avoid persistence of L. monocytogenesresistant strains in the dairy environment.

\section{Abbreviations}

BAC, benzalkonium chloride; CFU, colony-forming unit; DMSO, dimethyl sulfoxide; QAC, quaternary ammonium compound.

\section{Data Sharing Statement}

All data sets generated or analyzed during this study are included in the manuscript.

\section{Acknowledgments}

The authors extend their appreciation to the Taif University Researchers Supporting Program (project TURSP-2020/128), Taif University, Saudi Arabia.

\section{Author Contributions}

All authors contributed to data analysis, drafting or revising the article, have agreed on the journal to which the article will be submitted, gave final approval to the version to be published, and agree to be accountable for all aspects of the work. 


\section{Disclosure}

The authors declare that they have no conflicts of interest for this work.

\section{References}

1. Tabit FT. Contamination, prevention and control of Listeria monocytogenes in food processing and food service environments. In: Nyila MA editor. Listeria Monocytogenes. IntechOpen; 2018:71. doi:10.5772/intechopen.76132.

2. Ricci A, Allende A, Bolton D, et al.; EFSA Panel on Biological Hazards (BIOHAZ). Listeria monocytogenes contamination of ready-to-eat foods and the risk for human health in the EU. EFSA $J$. 2018;16(1):e05134. doi:10.2903/j.efsa.2018.5134

3. Liu D, Lawrence ML, Austin FW, Ainsworth AJ. A multiplex PCR for species-and virulence-specific determination of Listeria monocytogenes.. J Microbiol Methods. 2007;71(2):133-140. doi:10.1016/j. mimet.2007.08.007

4. Tirumalai PS, Prakash S. Expression of virulence genes by Listeria monocytogenes $\mathrm{J} 0161$ in natural environment. Braz J Microbiol. 2012;43(2):834-843. doi:10.1590/S1517-83822012000200050

5. Skowron K, Wałecka-Zacharska E, Grudlewska K, et al. Disinfectant susceptibility of biofilm formed by Listeria monocytogenes under selected environmental conditions. Microorganisms. 2019;7(9):280. doi:10.3390/microorganisms 7090280

6. Martínez-Suárez JV, Ortiz S, López-Alonso V. Potential impact of the resistance to quaternary ammonium disinfectants on the persistence of Listeria monocytogenes in food processing environments. Front Microbiol. 2016;7:638. doi:10.3389/fmicb.2016.00638

7. Aase B, Sundheim G, Langsrud S, Rørvik LM. Occurrence of and a possible mechanism for resistance to a quaternary ammonium compound in Listeria monocytogenes. Int J Food Microbiol. 2000;62(12):57-63. doi:10.1016/S0168-1605(00)00357-3

8. Müller A, Rychli K, Muhterem-Uyar M, et al. Tn 6188-a novel transposon in Listeria monocytogenes responsible for tolerance to benzalkonium chloride. PLoS One. 2013;8(10):e76835. doi:10.1371/ journal.pone.0076835

9. Yamamura A, Murai A, Takamatsu H, Watabe K. Antimicrobial effect of chemical preservatives on enterohemorrhagic Escherichia coli O157: H7. Health Sci J. 2000;46(3):204-208. doi:10.1248/ jhs.46.204

10. Bialonska D, Ramnani P, Kasimsetty SG, Muntha KR, Gibson GR, Ferreira D. The influence of pomegranate by-product and punicalagins on selected groups of human intestinal microbiota. Int J Food Microbiol. 2010;140(2-3):175-182. doi:10.1016/j.ijfoodmicro.20 10.03 .038

11. Aspri M, O’Connor PM, Field D, et al. Application of bacteriocinproducing Enterococcus faecium isolated from donkey milk, in the bio-control of Listeria monocytogenes in fresh whey cheese. Int Dairy J. 2017;73:1-9. doi:10.1016/j.idairyj.2017.04.008

12. Cox S, Turley GH, Rajauria G, Abu-Ghannam N, Jaiswal AK. Antioxidant potential and antimicrobial efficacy of seaweed (Himanthalia elongata) extract in model food systems. J Appl Phycol. 2014;26(4):1823-1831. doi:10.1007/S10811-013-0215-0

13. Pérez MJ, Falqué E, Domínguez H. Antimicrobial action of compounds from marine seaweed. Mar Drugs. 2016;14(3):52. doi: $10.3390 / \mathrm{md} 14030052$

14. Dussault D, Vu KD, Vansach T, Horgen FD, Lacroix M. Antimicrobial effects of marine algal extracts and cyanobacterial pure compounds against five foodborne pathogens. Food Chem. 2016;199:114-118. doi:10.1016/j.foodchem.2015.11.119

15. Norton DM, Braden CR. Foodborne Listeriosis. In: Ryser ET, Marth EH, editors. Listeria, Listeriosis and Food Safety. 3rd ed. Boca Raton: CRC Press Taylor \& Francis Group; 2007:305-356.
16. Akrami-Mohajeri F, Derakhshan Z, Ferrante M, et al. The prevalence and antimicrobial resistance of Listeria spp in raw milk and traditional dairy products delivered in Yazd, central Iran (2016). Food Chem Toxicol. 2018;114:141-144. doi:10.1016/j.fct.2018.02.006

17. Rahimi E, Ameri M, Momtaz H. Prevalence and antimicrobial resistance of Listeria species isolated from milk and dairy products in Iran. Food Control. 2010;21(11):1448-1452. doi:10.1016/j. foodcont.2010.03.014

18. World Health Organization. International Standards for Drinking Water. 3rd. Geneva: Plais de Nation; 1971.

19. Rendos JJ, Eberhart RJ, Kesler EM. Microbial populations of teat ends of dairy cows, and bedding materials. J Dairy Sci. 1975;58 (10):1492-1500. doi:10.3168/jds.S0022-0302(75)84740-0

20. Roberts D, Greenwood M. Practical Food Microbiology. 3rd ed. USA: Black well Publishing Ltd; 2003.

21. Singh P, Prakash A. Isolation of Escherichia coli, Staphylococcus aureus and Listeria monocytogenes from milk products sold under market conditions at Agra region. Acta Agric Slov. 2008;92(1):83-88.

22. Chye FY, Abdullah A, Ayob MK. Bacteriological quality and safety of raw milk in Malaysia. Food Microbiol. 2004;21(5):535-541. doi:10.1016/j.fm.2003.11.007

23. Deneer HG, Boychuk I. Species-specific detection of Listeria monocytogenes by DNA amplification. Appl Environ Microbiol. 1991;57 (2):606-609. doi:10.1128/AEM.57.2.606-609.1991

24. Dickinson JH, Kroll RG, Grant KA. The direct application of the polymerase chain reaction to DNA extracted from foods. Lett Appl Microbiol. 1995;20(4):212-216. doi:10.1111/j.1472-765X.1995.tb00430.x

25. Sambrook J, Fritsch EF, Montias T. Molecular Biology. In: Molecular Cloning. Laboratory Manual. 2nd ed. USA: Cold Spring Harbor Laboratory press; 1989:268.

26. BS EN 1276:1997. Chemical disinfectants and antiseptics - quantitative suspension test for the evaluation of bactericidal activity of chemical disinfectants and antiseptics used in food, industrial, domestic, and institutional areas - test method and requirements (phase 2, step 1). London, UK: British Standards Institute; 1997.

27. Russell AD. Neutralization procedures in the evaluation of bactericidal activity. In: Collins CH, Allwood MC, Bloomfield SF, Fox A, editors. Disinfectants: Their Use and Evaluation of Effectiveness. UK: Academic Press; 1981:45-59.

28. Espigares E, Bueno A, Fernández-Crehuet M, Espigares M. Efficacy of some neutralizers in suspension tests determining the activity of disinfectants. J Hosp Infect. 2003;55(2):137-140. doi:10.1016/ S0195-6701(03)00238-X

29. Edwards M, Hannifffy D, Heesch S, et al. Macroalgae Fact-sheets. Irish Seaweed Research Group; Ryan Institute, NUI Galway. 2012:40. Available at: http://www.seaweed.ie/irish_seaweed_con tacts/doc/FactSheets.pdf.

30. Aleem AA. The Marine Algae of Alexandria, Egypt. Egypt: Univ. Alexandria; 1993:139.

31. Ahmed AS, Diab HM, Alkahtani MA, et al. Molecular epidemiology of virulent $E$. coli among rural small scale dairy herds and shops: efficacy of selected marine algal extracts and disinfectants. Int J Environ Health Res. 2020;15:1-23. doi:10.1080/09603123.2020.1727422

32. Saber H, Alwaleed EA, Ebnalwaled KA, Sayed A, Salem W. Efficacy of silver nanoparticles mediated by Jania rubens and Sargassum dentifolium macroalgae; Characterization and biomedical applications. Egypt J Basic Appl Sci. 2017;4(4):249-255. doi:10.1016/j.ejbas.2017.10.006

33. El-Sheekh MM, Alwaleed EA, Kassem WM, Saber H. Antialgal and antiproliferative activities of the algal silver nanoparticles against the toxic cyanobacterium Microcystis aeruginosa and human tumor colon cell line. Environ Nanotechnol Monit Manag. 2020;14:100352. doi:10.1016/j.enmm.2020.100352

34. Zainol MI, Yusoff KM, Yusof MY. Antibacterial activity of selected Malaysian honey. BMC Complement Altern Med. 2013;13(1):129. doi:10.1186/1472-6882-13-129 
35. Abou-Donia SA. Egyption Domiati pickled cheese. NZJ Dairy Sci Technol. 1986;21:167.

36. Skandamis PN, Gounadaki AS, Geornaras I, Sofos JN. Adaptive acid tolerance response of Listeria monocytogenes strains under planktonic and immobilized growth conditions. Int $J$ Food Microbiol. 2012;159(2):160-166. doi:10.1016/j.ijfoodmicro.201 2.07.027

37. Angelidis AS, Papageorgiou DK, Tyrovouzis NA, Stoforos NG. Kinetics of Listeria monocytogenes cell reduction in processed cheese during storage. Food Control. 2013;29(1):18-21. doi:10.1016/j.foodcont.2012.05.062

38. Ivanek R, Gröhn YT, Wiedmann M. Listeria monocytogenes in multiple habitats and host populations: review of available data for mathematical modeling. Foodborne Pathog Dis. 2006;3(4):319-336. doi:10.1089/fpd.2006.3.319

39. Nightingale KK, Schukken YH, Nightingale CR, et al. Ecology and transmission of Listeria monocytogenes infecting ruminants and in the farm environment. Appl Environ Microbiol. 2004;70(8):44584467. doi:10.1128/AEM.70.8.4458-4467.2004

40. Shiwakoti S. Prevalence of Listeria monocytogenes in the pre-harvest environment; a landscape epidemiology approach. Master's Thesis. The Graduate Faculty of the North Dakota State University of Agriculture and Applied Science, USA; 2015.

41. Hellstrom S. Contamination routes and control of Listeria monocytogenes in food production. Ph.D. Thesis. Department of Food Hygiene and Environmental Health, Faculty of Veterinary Medicine, University of Helsinki, Finland; 2011.

42. Abd El Tawab AA, Maarouf AA, Mahdy ZA. Bacteriological and molecular studies of Listeria species in milk and milk products at ElKaliobia governorate. Benha Vet Medl J. 2015;29(2):170-181. doi:10.21608/bvmj.2015.31698

43. Nayak DN, Savalia CV, Kalyani IH, Kumar R, Kshirsagar DP. Isolation, identification, and characterization of Listeria spp. from various animal origin foods. Vet World. 2015;8(6):695. doi:10.14202/vetworld.2015.695-701

44. McDonnell G, Russell AD. Antiseptics and disinfectants: activity, action, and resistance. Clin Microbiol Rev. 1999;12:147-179. PubMed: 9880479.

45. Heir E, Lindstedt B-A, Røtterud O-J, Vardund T, Kapperud G, Nesbakken T. Molecular epidemiology and disinfectant susceptibility of Listeria monocytogenes from meat processing plants and human infections. Int J Food Microbiol. 2004;96(1):85-96. doi:10.1016/j. ijfoodmicro.2004.03.014

46. Mullapudi S, Siletzky RM, Kathariou S. Heavy-metal and benzalkonium chloride resistance of Listeria monocytogenes isolates from the environment of Turkey-processing plants. Appl Environ Microbiol. 2008;74(5):1464-1468. doi:10.1128/AEM.02426-07

47. Møretrø T, Schirmer BC, Heir E, Fagerlund A, Hjemli P, Langsrud S. Tolerance to quaternary ammonium compound disinfectants may enhance growth of Listeria monocytogenes in the food industry. Int $J$ Food Microbiol. 2017;241:215-224. doi:10.1016/j. ijfoodmicro.2016.10.025

48. Ebner R, Stephan R, Althaus D, Brisse S, Maury M, Tasara T. Phenotypic and genotypic characteristics of Listeria monocytogenes strains isolated during 2011-2014 from different food matrices in Switzerland. Food Control. 2015;57:321-326. doi:10.1016/j. foodcont.2015.04.030

49. Müller A, Rychli K, Zaiser A, Wieser C, Wagner M, Schmitz-Esser S. The Listeria monocytogenes transposon $\mathrm{Tn} 6188$ provides increased tolerance to various quaternary ammonium compounds and ethidium bromide. FEMS Microbiol Lett. 2014;361(2):166-173. doi:10.1111/1574-6968.12626
50. Best M, Kennedy ME, Coates F. Efficacy of a variety of disinfectants against Listeria spp.. Appl Environ Microbiol. 1990;56(2):377-380. [PubMed: 2106285]. doi:10.1128/AEM.56.2.377-380.1990

51. Buffet-Bataillon S, Tattevin P, Bonnaure-Mallet M, Jolivet-Gougeon A. Emergence of resistance to antibacterial agents: the role of quaternary ammonium compounds - a critical review. Int J Antimicrob Agents. 2012;39(5):381-389. doi:10.1016/j.ijantimicag.2012.01.011

52. Ortiz S, López V, Martínez-Suárez JV. The influence of subminimal inhibitory concentrations of benzalkonium chloride on biofilm formation by Listeria monocytogenes. Int $J$ Food Microbiol. 2014;189:106-112. doi:10.1016/j.ijfoodmicro.2014.08.007

53. Ö E, Taş B. Antibacterial and antifungal effects of some marine algae. Kafkas Univ Vet Fak Derg. 2011;17(SupplA):S121-S124.

54. Karima S, Fatiha B. Study of the antimicrobial activity of four algerian marin algae species. In: Mendez-Vilas A editor. Microbes in Applied Research: Current Advances and Challenges. World Scientific; 2012:578-581. doi:10.1142/9789814405041_0118.

55. Saleh B, Haj-Mahmoud N, Al-Mariri A. In-vitro Inhibitory effect of algae crude extracts against some gram-positive bacterial pathogens. Int J Pharm Life Sci. 2013;9(11\&12):5991-6001.

56. Rao BV, Boominathan M. Antibacterial activity of silver nanoparticles of seaweeds. Am J Adv Drug Delivery. 2015;3:296-307.

57. Nagappan T, Vairappan CS. Nutritional and bioactive properties of three edible species of green algae, genus Caulerpa (Caulerpaceae). J Appl Phycol. 2014;26(2):1019-1027. doi:10.1007/s10811-013-0147-8

58. Patel NB, Patel LN, Patel MV, Prajapati ND, Kalasariya HS. In silico approach to check the effect of red algae gracillaria corticata on bacterial food pathogen Listeria monocytogenes. Int J Multidiscip Educ Res. 2020;9(1):90-102.

59. Cokal Y, Dagdelen A, Cenet O, Gunsen U. Presence of L. monocytogenes and some bacterial pathogens in two Turkish traditional foods, Mihalic cheese and Hosmerim dessert. Food Control. 2012;26 (2):337-340. doi:10.1016/j.foodcont.2012.01.058

60. Rocha PRDA, Lomonaco S, Bottero MT, et al. Ruminant rhombencephalitis-associated Listeria monocytogenes strains constitute a genetically homogeneous group related to human outbreak strains. Appl Environ Microbiol. 2013;79(9):3059-3066. doi:10.1128/ AEM.00219-13

61. El Shinaway SH, Meshref AMS, Zeinhom MMA, Hafez A. The survival of Listeria monocytogenes in yoghurt and ice cream. $J$ Vet Med Res. 2017;24(2):235-246. doi:10.21608/jvmr.2017.43288

62. Güner A. In vitro risk assessment of Padina pavonica (Linnaeus) (Brown algae). Food Health. 2020;7(1):31-38. doi:10.3153/FH21004

63. Al-Enazi NM, Awaad AS, Zain ME, Alqasoumi SI. Antimicrobial, antioxidant and anticancer activities of Laurencia catarinensis, Laurencia majuscula and Padina pavonica extracts. Saudi Pharml J. 2018;26(1):44-52. doi:10.1016/j.jsps.2017.11.001

64. Samelis J, Kakouri A, Rogga KJ, Savvaidis IN, Kontominas MG. Nisin treatments to control Listeria monocytogenes post-processing contamination on Anthotyros, a traditional Greek whey cheese, stored at $4{ }^{\circ} \mathrm{C}$ in vacuum packages. Food Microbiol. 2003;20(6):661-669. doi:10.1016/S0740-0020(03)00018-2

65. Cho EJ, Nam ES, Park SI. Keeping quality and sensory properties of drinkable yoghurt with added Chlorella extract. Korean J Food Nutr. 2004;17:128-132.

66. Jeon JK. Effect of Chlorella addition on the quality of processed cheese. J Korean Soc Food Sci Nutr. 2006;35:373-377. doi:10.3746/ jkfn.2006.3.5.3.373 


\section{Publish your work in this journal}

Infection and Drug Resistance is an international, peer-reviewed openaccess journal that focuses on the optimal treatment of infection (bacterial, fungal and viral) and the development and institution of preventive strategies to minimize the development and spread of resistance. The journal is specifically concerned with the epidemiology of antibiotic resistance and the mechanisms of resistance development and diffusion in both hospitals and the community. The manuscript management system is completely online and includes a very quick and fair peerreview system, which is all easy to use. Visit http://www.dovepress.com/ testimonials.php to read real quotes from published authors.

Submit your manuscript here: https://www.dovepress.com/infection-and-drug-resistance-journal 